\title{
Bioindicators of climate and trophic state in lowland and highland aquatic ecosystems of the Northern Neotropics
}

\author{
Liseth Pérez*1,2, Julia Lorenschat ${ }^{1}$, Julieta Massaferro ${ }^{3}$, Christine Pailles ${ }^{4}$, Florence Sylvestre ${ }^{4}$,
} Werner Hollwedel ${ }^{5}$, Gerd-Oltmann Brandorff ${ }^{6}$, Mark Brenner ${ }^{7}$, Gerald Islebe ${ }^{8}$, María del Socorro Lozano $^{2}$, Burkhard Scharf ${ }^{1} \&$ Antje Schwalb ${ }^{1}$

1. Institut für Geosysteme und Bioindikation, Technische Universität Braunschweig, Langer Kamp 19c, 38106 Braunschweig, Germany; 1.perez@tu-bs.de, j.lorenschat@tu-bs.de, burkhard.w.scharf@t-online.de, antje.schwalb@tu-bs.de

2. Instituto de Geología, Universidad Nacional Autónoma de México (UNAM), Ciudad Universitaria, 04510, Distrito Federal, México; lcpereza@geologia.unam.mx,mslozano@unam.mx

3. CENAC-APN, CONICET, San Martin 24, 8400, Bariloche, Argentina; julimassaferro@hotmail.com

4. CEREGE, Université Aix-Marseille, CNRS, IRD, Europôle méditerranéen de l'Arbois, BP 80, 13545 Aix-enProvence cedex 4, France; pailles@cerege.fr, sylvestre@cerege.fr

5. Oldenburger Strasse 16A, 26316, Varel, Germany; whollwedel@freenet.de

6. Georg-Gröning-Str. 29A, 28209, Bremen, Germany; gobrandorf@aol.com

7. Department of Geological Sciences \& Land Use and Environmental Change Institute, University of Florida, Gainesville, 32611, Florida, USA; brenner@ufl.edu

8. Herbario, El Colegio de la Frontera Sur (ECOSUR), Unidad Chetumal, Av. del Centenario 424, 77000, Chetumal, Quintana Roo, México; gislebe@ecosur.mx

* Corresponding author

Received 29-V-2012. Corrected 02-IX-2012. Accepted 04-X-2012.

\begin{abstract}
Chironomids, diatoms and microcrustaceans that inhabit aquatic ecosystems of the Northern Neotropics are abundant and diverse. Some species are highly sensitive to changes in water chemical composition and trophic state. This study was undertaken as a first step in developing transfer functions to infer past environmental conditions in the Northern lowland Neotropics. Bioindicator species abundances were related to multiple environmental variables to exploit their use as environmental and paleoenvironmental indicators. We collected and analyzed water and surface sediment samples from 63 waterbodies located along a broad trophic state gradient and steep gradients of altitude ( 0-1560m.a.s.1.) and precipitation ( $400-3200 \mathrm{~mm} / \mathrm{y})$, from NW Yucatán Peninsula (Mexico) to southern Guatemala. We related 14 limnological variables to relative abundances of 282 diatom species, 66 chironomid morphospecies, 51 species of cladocerans, 29 non-marine ostracode species and six freshwater calanoid copepods. Multivariate statistics indicated that bicarbonate is the strongest driver of chironomid and copepod distribution. Trophic state is the second most important factor that determines chironomid distribution. Conductivity, which is related to the precipitation gradient and marine influence on the Yucatán Peninsula, is the main variable that shapes diatom, ostracode and cladoceran communities. Diatoms, chironomids and cladocerans displayed higher diversities $(\mathrm{H}=2.4-2.6)$ than ostracodes and copepods $(\mathrm{H}=0.7$ 1.8). Species richness and diversity were greater at lower elevations $(<450$ m.a.s.1.) than at higher elevations in Guatemala. Distribution and diversity of bioindicators are influenced by multiple factors including altitude, precipitation, water chemistry, trophic state and human impact. Rev. Biol. Trop. 61 (2): 603-644. Epub 2013 June 01 .
\end{abstract}

Key words: microcrustaceans, chironomid, diatom, aquatic ecosystems, bioindicators, Northern Neotropics, autecology, diversity. 
Natural and anthropogenic factors influence physical and chemical lake variables and aquatic biota, especially environmentally sensitive phytoplankton, phytobenthos, zooplankton and zoobenthos communities. Such factors include water extraction, pollution, eutrophication, flow modification, changes in water level, habitat degradation, climate warming, and changes in evaporation and precipitation (Dudgeon et al. 2006). Continental waterbodies are some of the most endangered ecosystems in the world (Sala et al. 2000), especially those in developing countries (Pérez et al. 2011a). Waterbodies in the Northern Neotropics are important resources for local inhabitants. They provide drinking water, sites for recreation, navigation, and habitat for both aquatic and terrestrial fauna and flora (Dudgeon et al. 2006). Despite their importance, there have been few limnological or ecological studies of these ecosystems, especially in Guatemala and Belize (Pérez et al. 2011a). Many local inhabitants around the largest lakes in Guatemala, Lakes Izabal, Petén Itzá, Amatitlán and Atitlán, rely on local fisheries for subsistence and to generate income in local markets. Such activities have affected the trophic state of these lakes. Lake Petén Itzá, located in the Maya Biosphere Reserve in Northern Guatemala still displays high diversity of aquatic bioindicators, but cultural eutrophication in the lake has increased during the last few decades (Rosenmeier et al. 2004, Pérez et al. 2010a), putting many species at risk. Lake Amatitlán is highly productive and has suffered from cultural eutrophication for decades (Pérez et al. 2011a). Aquatic bioindicator diversities in the lake are probably low because of the hypereutrophic conditions. Cyanobacteria dominate the phytoplankton community in the lake, and high rates of decomposition lead to hypoxic or anoxic conditions in deep waters. Cyanobacteria blooms in the lake produce toxins that can be dangerous to humans if present in high concentrations (Pérez et al. 2011a).

Aquatic organisms that are sensitive to changes in water chemical composition, pollution and trophic state, i.e. aquatic bioindicators such as diatoms, chironomids and microcrustaceans, are frequently used to track environmental change. Diatoms are generally a dominant group in the phytoplankton, whereas cladocerans, copepods and ostracodes are typically the main zooplankters in fresh waters (Dole-Olivier et al. 2000, Walseng et al. 2006). Diatoms are unicellular golden-brown algae (Bacillariophyta) characterized by silica shells (frustules) that are well preserved in lake sediments. Diatoms live in planktonic and benthic habitats (Battarbee et al. 2001). Chironomids are non-biting midges (Insecta: Diptera) and are frequently the most abundant group of aquatic insects in fresh waters. Chironomids are true flies, but they spend most of their life cycle (egg, larva, pupa) in aquatic habitats (Armitage et al. 1995). They are ubiquitous inhabitants of Neotropical aquatic ecosystems. Nevertheless, there have been few studies in the region concerning their taxonomy and autecology (Pérez et al. 2010a). Microcrustaceans such as ostracodes, cladocerans and copepods are important organisms in limnological and paleolimnological studies. Ostracodes are typically $<3 \mathrm{~mm}$ long. The two valves that enclose the body are composed of low-Mg calcite (Meisch 2000). Similar to ostracodes, cladocerans are small $(0.2-2.5 \mathrm{~mm})$. Limbs and a postabdomen extend from a ventral opening in the carapace, facilitating locomotion and feeding (Dole-Olivier et al. 2000). Ostracode valves and cladoceran exoskeletons preserve well in lake sediments. Body parts of freshwater copepods $(<2.0 \mathrm{~mm}$ long), however, are poorly preserved. Nevertheless, sacs with resting eggs of some copepod species are robust and well preserved in late Quaternary lake sediments (Bennike 1998). Microcrustaceans, diatoms and chironomids are the main food sources for many aquatic macroinvertebrates and for vertebrates such as fish. They are key components of the food web in lake ecosystems and therefore of great ecological and economic value (Cohen 2003, O'Sullivan \& Reynolds 2004). Impacts on these communities from pollution, changes in lake trophic state or climate, can have dramatic consequences for fish populations (Moss 
et al. 2003). Microcrustaceans, diatoms and chironomids are widely distributed, can rapidly colonize new habitats (Cohen 2003, Hausmann \& Pienitz 2007) and share characteristics that make them useful as bioindicators and paleoindicators: (1) their well preserved remains in lake sediments can be identified to genus, and sometimes to species level, (2) they are often abundant, (3) they are highly sensitive to environmental changes, (4) they have short life cycles and communities thus respond quickly to environmental changes.

Consistent taxonomy, along with information on species autecology and the factors that affect species distributions and diversity, are indispensable to ensure that inferences from bioindicators, whether in modern or paleoenvironmental contexts, are valid. There have been few paleolimnological studies using bioindicators in remote tropical areas, in large part because of the paucity of autecological data. Detailed bioindicator analysis, coupled with information on physical and chemical attributes of aquatic ecosystems, is required to fully exploit the utility of such bioindicator taxa. These taxonomic groups are highly sensitive to environmental changes, such as shifts in salinity, conductivity or ionic concentration (Fritz et al. 1991, Smith 1993, Pérez et al. 2011b), total phosphorus concentration (Hausmann \& Kienast 2006), lake level (Sylvestre 2002), air temperature (Walker et al. 1997, Brooks \& Birks 2001), $\mathrm{pH}$ and organic matter concentration (Rosén et al. 2000), and changes in precipitation and trophic state (Massaferro et al. 2004, Pérez et al. 2010a).

There has been little research on the autecology of lacustrine organisms in the Northern Neotropics. Most studies have focused on taxonomy and biogeography. Studied groups include cladocerans (Elías-Gutiérrez et al. 2006, 2008) and copepods (Suárez-Morales \& Elías-Gutiérrez 2000, Suárez-Morales \& Reid 2003). Pérez et al. (2010a,b,c, 2011b) recently conducted studies on the freshwater ostracode fauna of the Yucatán Peninsula and surrounding areas. There are, however, few studies on diatoms and chironomids. This study presents information on chironomid, diatom, cladoceran, copepod and ostracode taxa from 63 waterbodies in the Northern Neotropics, along with associated environmental data. Our objective was to determine the factors that govern the distributions of these bioindicators so they could be used to infer late Quaternary environmental conditions and climate on the Yucatán Peninsula, Guatemala and Belize. In this study, we (1) present an inventory of the main species that inhabit aquatic ecosystems of the Northern Neotropics, (2) display ecological information from the studied waterbodies, (3) evaluate relationships between bioindicator relative abundances and environmental variables, (4) identify areas with high species richness and diversity that could be of conservation interest in this zoogeographic province and (5) develop a basis for transfer functions that can be applied in paleolimnological studies to infer past environmental variables such as water chemical composition and lake level. Ultimately, these transfer functions will be applied to fossil assemblages in long sediment cores retrieved from Lago Petén Itzá, Guatemala and other waterbodies in the Northern Neotropics to infer past environmental variables.

\section{MATERIALS AND METHODS}

Study site: The Yucatán Peninsula (Mexico, Guatemala and Belize, Fig. 1) and surrounding areas are rich in aquatic ecosystems that have different origins (tectonic, volcanic, karstic) and possess diverse water chemical composition. Chemical characteristics of waterbodies are mainly influenced by bedrock geology, climate and saltwater intrusion at coastal sites (Pérez et al. 2011a). The Yucatán Peninsula (Fig. 1) is a marine carbonate platform. The region is of interest to ecologists and paleoecologists alike, because it displays steep, increasing NW-S precipitation ( 400$3200 \mathrm{~mm} / \mathrm{y})$ and altitude ( 0-1560m.a.s.1.) gradients (Pérez et al. 2011a). A dry season (January-May) and a rainy season (JuneOctober) characterize the Yucatán Peninsula and surrounding areas. Short-duration showers 


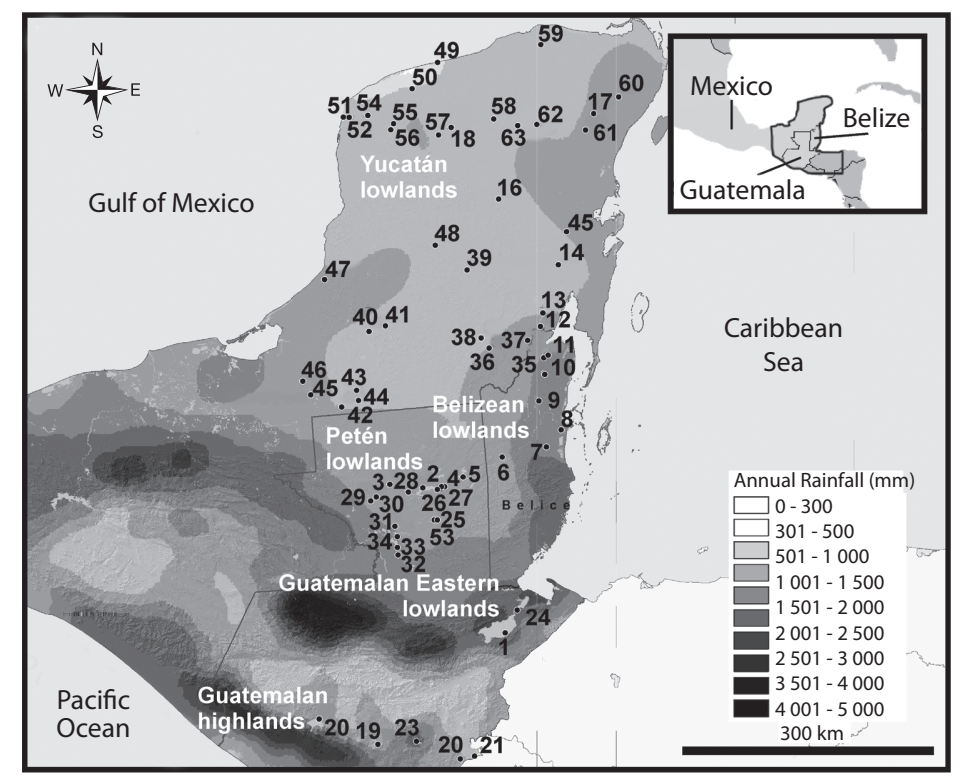

Fig. 1. Map of the 63 sampled lakes, "cenotes", coastal waterbodies, rivers, wetland and ponds on the Yucatán Peninsula. Studied regions included the Guatemalan highlands, eastern lowlands, Petén lowlands, Belize lowlands and Yucatán lowlands. Full names, ID and ID-Nr. (number on this figure) of the sampled aquatic ecosystems can be found in tables 1a (lakes) and $1 \mathrm{~b}$ ("cenotes", coastal lagoons, rivers, wetland and ponds). Gray scale shading indicates the steep, increasing NW-S precipitation gradient on the study area.

usually occur from November to December (Schmitter-Soto et al. 2002). Most of the study area is located in a dry tropical climate zone that is rich in aquatic ecosystems and displays high aquatic biodiversity (Lutz et al. 2000, Pérez et al. 2011a).

Sampling and habitat characterization: Two fieldtrips were carried out in the Yucatán Peninsula (Mexico), Guatemala and Belize $\left(14^{\circ} 13^{\prime} 00^{\prime \prime}-21^{\circ} 25^{\prime} 00^{\prime \prime} \mathrm{N}\right.$ and $87^{\circ} 20^{\prime} 00^{\prime \prime}$ $91^{\circ} 03^{\prime} 00^{\prime \prime} \mathrm{W}$ ) in 2005-2006 and 2008. A single sampling was carried out for each lake. Chironomids, diatoms and microcrustaceans (cladocerans, copepods, ostracodes) were collected from 63 aquatic ecosystems (Fig. 1, Table 1a, b). These ecosystems included deep (10-340m) and shallow $(<10 \mathrm{~m})$ lakes (Table 1a), "cenotes" (sinkholes), coastal lagoons, ponds, rivers, and wetlands (Table 1b). Surface sediment samples (lake deepest point, littoral zones, other water depths) were retrieved using an
Ekman grab. Ostracodes and cladocerans that live in macrophyte-rich littoral zones were collected with $250 \mu \mathrm{m}$ and $100 \mu \mathrm{m}$-mesh hand nets, respectively. Physical and chemical variables and the chemical and isotopic composition of lake waters were studied to better characterize the habitat. Water samples were collected from at least three depths above the lake's deepest point (surface, mid-depth and bottom). Only surface waters near the shore were collected in smaller water bodies (ponds, rivers and wetlands). Water temperature, dissolved oxygen, $\mathrm{pH}$ and conductivity in surface waters were measured in situ using a WTW Multi Set 350i. Most measurements were done at midday. Water samples were collected in duplicate for laboratory analysis of $\mathrm{Ca}, \mathrm{Na}, \mathrm{Mg}, \mathrm{K}, \mathrm{Cl}$, $\mathrm{HCO}_{3}, \mathrm{SO}_{4}$, and for $\delta^{18} \mathrm{O}$ and $\delta^{13} \mathrm{C}_{\mathrm{DIC}}$ analysis. $\delta^{18} \mathrm{O}$ values were used as an indicator of the balance between evaporation and precipitation and $\delta^{13} \mathrm{C}$ values as a productivity proxy (Schwalb 2003). Cations were measured using 


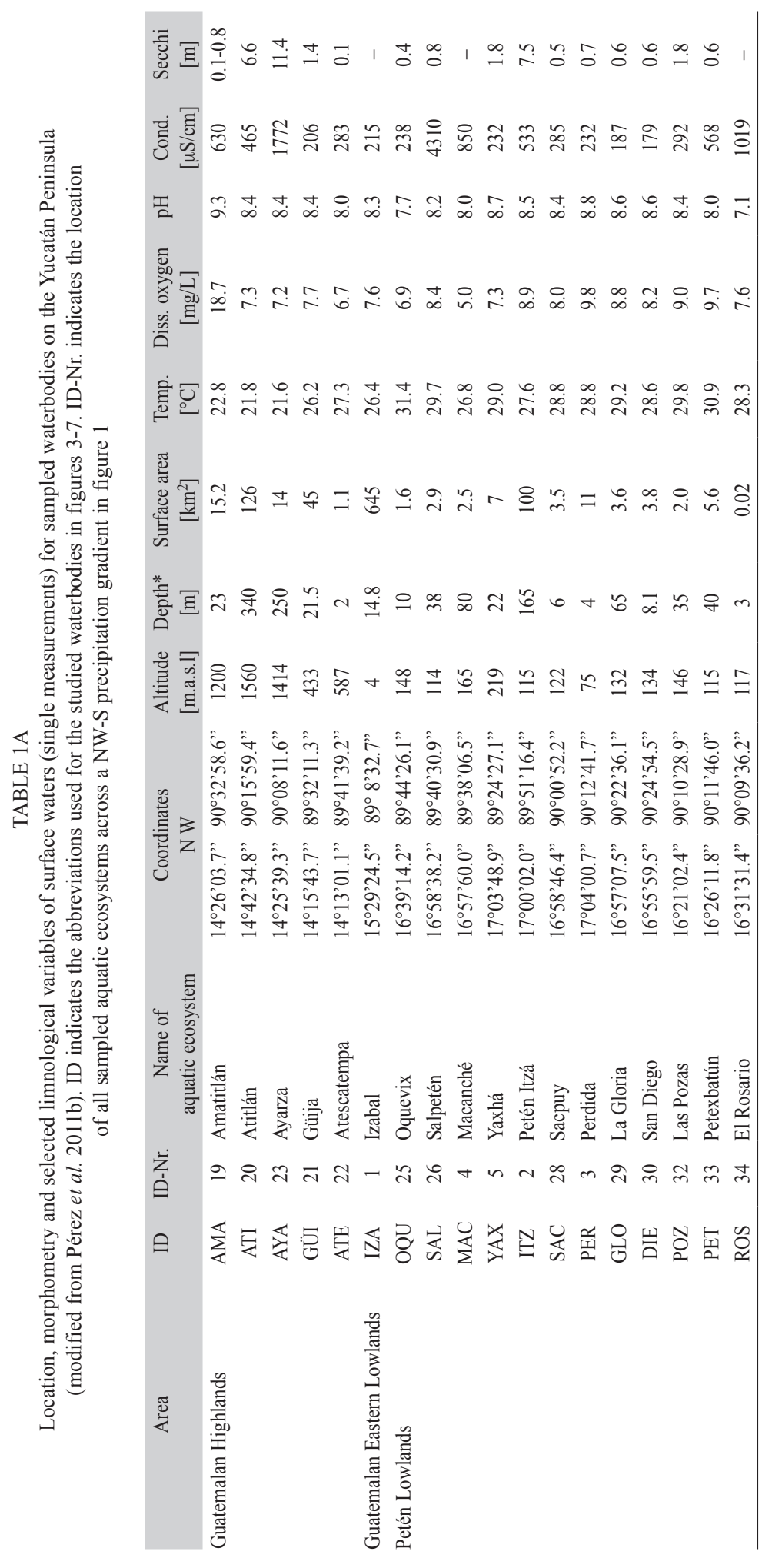




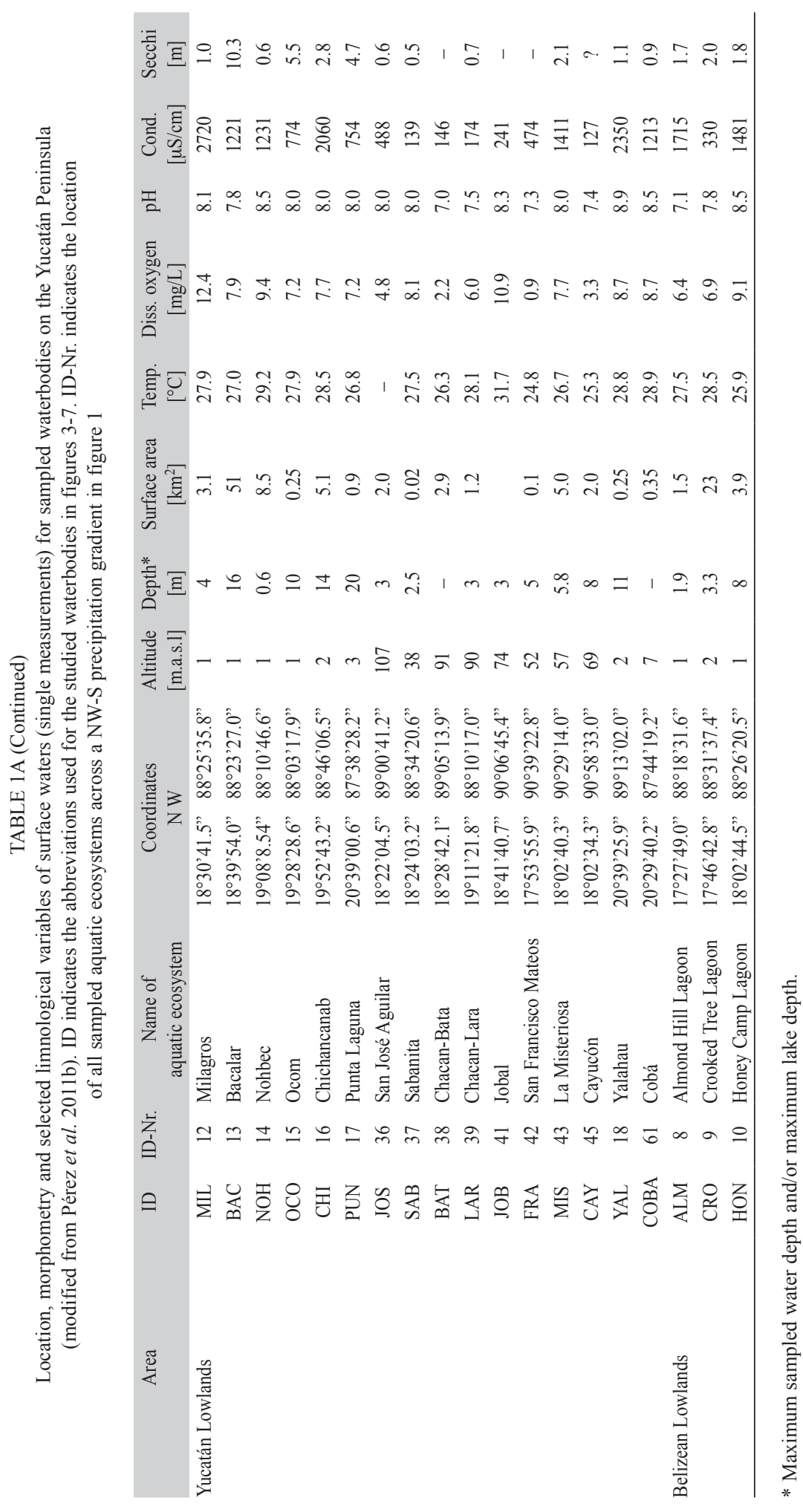




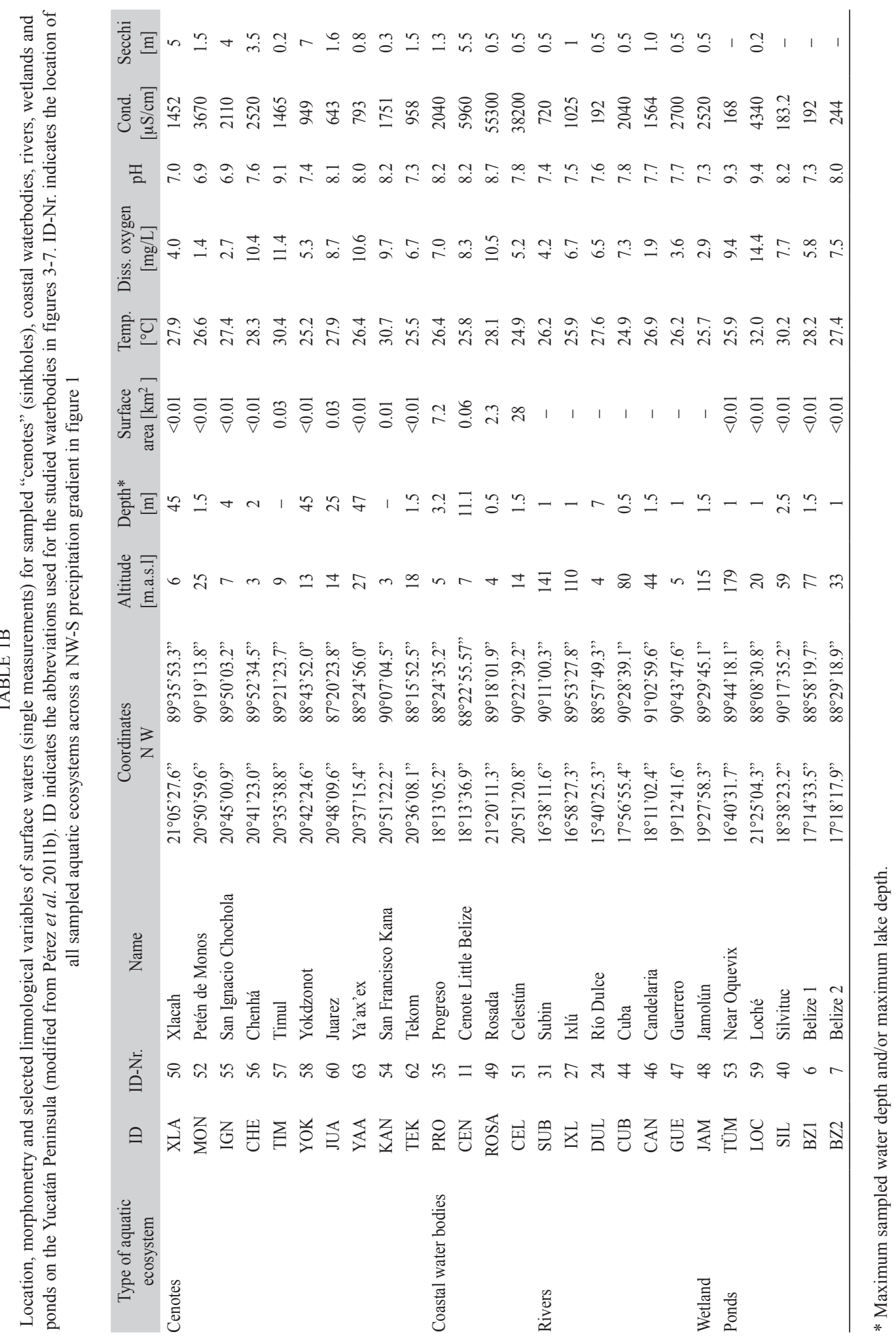


an ICP-OES Jobin Yvon JY 50 P Spectrometer. Bicarbonate was determined by titration with $0.1 \mathrm{~N} \mathrm{HCl}$. Anions were measured using a 761 Compact IC Methrom at the Institut für Umweltgeologie, Technische Universität Braunschweig, Germany. Carbon and oxygen isotopes in waters were analyzed on a VG/ Micromass PRISM Series II isotope ratio mass spectrometer and a Finnigan-MAT DeltaPlus $\mathrm{XL}$ isotope ratio mass spectrometer with a GasBench II universal on-line gas preparation device at the University of Florida, USA.

Bioindicator analysis: Surface sediments ( $\sim 3 g$ wet sediment) for chironomid analysis were (1) deflocculated in $10 \% \mathrm{KOH},(2)$ heated to $70^{\circ} \mathrm{C}$ for 10 minutes, (3) heated in water to $90^{\circ} \mathrm{C}$ for 20 minutes, and (4) sieved using $212 \mu \mathrm{m}$ and $95 \mu \mathrm{m}-\mathrm{mesh}$ sieves. Chironomid head capsules were extracted from samples using a Bogorov sorting tray and fine forceps. Head capsules were slide-mounted in Euparal, identified, counted and photographed. Identification followed Pérez et al. (2010a). We identified taxa to the morphospecies level because taxonomic data are generally lacking for the Northern Neotropics.

Sediment samples for diatom analysis were treated with hot concentrated $\mathrm{HNO}_{3}$, then with $33 \% \mathrm{H}_{2} \mathrm{O}_{2}$, followed by successive rinsing and decanting with distilled water. Sub-samples of the homogenized solution were diluted by adding distilled water and were left to settle onto coverslips until dry. The coverslips were fixed onto glass slides with Naphrax ${ }^{\circledR}$ mountant (refraction index=1.73). Counting was performed generally on three slides using a Nikon NS600 microscope at 1000x magnification. The total number of valves counted per sample varied from 50 in nearly sterile samples to $>1000$ in rich samples. Diatom identification and taxonomy followed Krammer \& LangeBertalot (1986, 1988, 1991a, 1991b) revised by the nomenclature of E. Fourtanier \& J.P. Kociolek (on-line version of the Catalog of Diatom Names: http://research.calacademy.org).

Surface sediments were initially analyzed for cladocerans using low magnification on a light microscope. Remains were isolated, identified, and counted and specimens were kept in small vials filled with 3-4\% formaldehyde solution. Several drops of glycerin were added to all vials to prevent desiccation. Permanent preparations of peculiar species were prepared for detailed microscopic observation to facilitate identification. We used polyvinyl lactophenol or Hydro-Matrix ${ }^{\circledR}$ as mounting media. Species were identified using the works of Korovchinsky (1992), Smirnov (1992, 1996), Lieder (1996), Flössner (2000), Kotov \& Stifler (2006), Elías-Gutiérrez et al. (2008) and Van Damme et al. (2011). Calanoid copepods that live in open waters and littoral zones were sampled with a plankton net (100- $\mu \mathrm{m}$ mesh), preserved with $10 \%$ formalin, and identified and counted under a dissecting microscope. Literature used for taxonomic identification included Bowman (1996), Gutiérrez-Aguirre \& Suárez-Morales (2000), Suárez-Morales \& Elías-Gutiérrez (2000, 2001), and ElíasGutiérrez et al. (2008). The details of the method used for ostracode analysis is in Pérez et al. (2011b). At least 100 adult ostracode valves were extracted from $50 \mathrm{~mL}$ of wet surface sediment. Samples were wet-sieved using

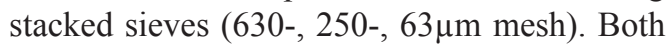
hard and soft parts were analyzed and used for identification to species level when possible. Identification followed Furtos (1933, 1936a, b), Brehm (1939), Keyser (1976), and Pérez et al. (2010a, b, c, 2011 b). Samples are stored at the Institut für Geosysteme und Bioindikation, Braunschweig, Germany. All bioindicator data are presented as relative abundances.

Species richness (S), i.e. the total number of species, and biodiversity, i.e. the Shannon Wiener Index (H) (Krebs 1989), were determined for each taxonomic group (chironomids, diatoms, cladocerans, copepods and ostracodes) in all waterbodies. Multivariate analysis was used to characterize species autecology by relating species relative abundances to water variables. Prior to statistical analysis, 14 environmental variables (water depth, water temperature, conductivity, dissolved oxygen (DO), $\mathrm{pH}, \delta^{18} \mathrm{O}, \delta^{13} \mathrm{C}_{\text {DIC }}, \mathrm{Ca}, \mathrm{K}, \mathrm{Mg}, \mathrm{Na}, \mathrm{Cl}$, 
$\mathrm{HCO}_{3}, \mathrm{SO}_{4}$ ) from all waterbodies were standardized (x-mean/st dev) and species relative abundances were log-transformed. Rare species, i.e. those present in $<3$ waterbodies, and samples containing few or no specimens, were excluded from analysis. Species included in the multivariate analysis are shown in bold in tables 2, 3 and 4. Thirty-eight chironomid, 97 diatom, 32 cladoceran, 3 copepod and 17 ostracode species were included in the statistical analysis. Correlations between environmental factors and the relative abundance of organisms were explored using Pearson correlation, which allowed up to seven environmental variables to be included in statistical analysis. Seven environmental variables were forward selected for statistical analysis of chironomids $(\mathrm{DO}, \mathrm{pH}$, temperature, conductivity, $\mathrm{HCO}_{3}, \delta^{13} \mathrm{C}$, water depth) and diatoms ( $\mathrm{DO}, \mathrm{pH}$, temperature, conductivity, $\delta^{13} \mathrm{C}, \delta^{18} \mathrm{O}$, water depth), four for cladocera (DO, temperature, $\mathrm{HCO}_{3}$, conductivity), and six for copepods (temperature, $\mathrm{HCO}_{3}$, $\mathrm{Na}, \mathrm{Cl}, \delta^{18} \mathrm{O}$, water depth) and ostracodes (temperature, $\mathrm{pH}, \mathrm{HCO}_{3}, \mathrm{Na}$, conductivity, water depth). Forward selection of the environmental variables followed Hausmann \& Kienast (2006) and Mischke et al. (2007).

Detrended Correspondence Analysis (DCA) and Canonical Correspondence Analysis (CCA) were used to relate counts (relative abundance) of chironomids, diatoms, cladocerans and ostracodes to environmental variables, whereas Redundancy Analysis (RDA) was used for copepod counts. This was accomplished using Canoco for Windows 4.55 (Ter Braak \& Šmilauer 2002). We first estimated the length of environmental gradients using a DCA and then used a CCA and RDA to discern the environmental factors that control bioindicator distributions in the study area. Generally, if a gradient is short ( $<3 \mathrm{SD})$, a linear model should be used, whereas with larger gradients ( $>4 \mathrm{SD}$ ), a unimodal model is recommended, because the approximation using the linear function is poor (Lepš \& Šmilauer 2003).

\section{RESULTS}

We collected 66 chironomid species and morphospecies belonging to the subfamilies Chironominae, Orthocladiinae and Tanypodinae (Table 2), 282 diatom species that belong to the orders Centrales and Pennales (Table 3), 51 cladoceran species belonging to the orders Anompoda and Ctenopoda, six copepod species (Calanoida), and 29 ostracode species (Podocopina, Table 4). Photographs of selected species are shown in figure 2 . Figures 3, 4, 5 and 6 display the relative abundances and altitude ranges of the aquatic bioindicators.

Chironomids: Figures 3 a, b, c display the relative abundances of the most common chironomid morphospecies, i.e. $>10$ individuals per waterbody and present in $>2$ aquatic ecosystems. The dominant tribe was Chironomini and consisted of 32 morphospecies (Table 2). Widely distributed taxa, i.e. present in $>15$ aquatic environments, included Cladotanytarsus sp.1, Chironomus anthracinus, Cladopelma sp., Dicrotendipes sp., Goeldochironomus sp., Micropsectra sp., Parachironomus sp., Paratanytarsus sp.1, Polypedilum sp. and Polypedilum sp. 2. Chironomus anthracinus, Dicrotendipes sp., Goeldochironomus sp. and Labrundina sp. had the highest relative abundances in most aquatic environments. Most chironomid species were collected at lower elevations ( $<450 \mathrm{~m}$ a.s.1.). Only 15 species were collected in aquatic ecosystems in the Guatemalan highlands. Dominant species in highland lakes were Apedilum sp., Apsectrotanypus sp. and Chironomus anthracinus. Chironomus anthracinus dominated the chironomid community in hypereutrophic Lake Amatitlán, Southern Guatemala. Chironomids inhabiting mainly the Petén lowlands were Stempellina sp. and Coelotanypus/Clinotanypus. Chironomus plumosus was the dominant species in Progreso Lagoon, Belize, and Cladopelma sp. was collected in all studied aquatic ecosystems 
TABLE 2

Chironomids (family Chironomidae; $\mathrm{n}=66$ ) found in aquatic ecosystems in the Northern Neotropics. Species are ordered alphabetically within subfamilies and tribes. Species codes in bold $(n=38)$ were taxa included in multivariate analysis. Code indicates the species abbreviations used in Figures 3a,b,c and 8. A number or letter was designated along with the genus to identify different morphospecies. For the ecosystem studies see tables $1 \mathrm{~A}, \mathrm{~B}$

\begin{tabular}{|c|c|c|c|}
\hline Taxa & Code & & \\
\hline Subfamily Chironominae & & Cladotanytarsus sp. 2 & CLA2 \\
\hline Tribe Chironomini & & Micropsectra sp. & MICR \\
\hline Apedilum sp. & APED & Tanytarsini A & TANA \\
\hline Axarus sp. & AXAR & Tanytarsini C & TANC \\
\hline Beardius sp. & BEAR & Tanytarsini D & TAND \\
\hline Brundinella sp. & BRUN & Tanytarsini F & TANF \\
\hline Chironomus anthracinus Zetterstedt 1860 & CHAN & Tanytarsini J & TANJ \\
\hline Chironomus plumosus Linnaeus 1758 & CHPL & Tanytarsini K & TANK \\
\hline Cladopelma sp. & CLAD & Paratanytarsus sp. 1 & PAR1 \\
\hline Corynocera ambigua Zetterstedt 1838 & CORC & Paratanytarsus sp. 2 & PAR2 \\
\hline Corynocera olivieri type & CORO & Tribe Pseudochironomini & \\
\hline Cryptochironomus sp. & CRYP & Pseudochironomus sp. & PSEU \\
\hline Dicrotendipes sp. & DICR & Subfamily Orthocladiinae & \\
\hline Einfeldia $\mathrm{sp}$. & EINF & Corynoneura sp. & CORY \\
\hline Endochironomus sp. & ENDO & Cricotopus spp. & CRIC \\
\hline Endotribelos sp. & ENTR & Eukiefferiella $\mathrm{sp}$. & EUKI \\
\hline Glyptotendipes sp.1 & GLEN & Limnophies sp. & LIMN \\
\hline Glyptotendipes sp.2 & GLYP & Mesosmittia sp. & MESS \\
\hline Goeldochironomus sp. & GOEL & Parakiefferiella fennica Tuiskunen 1986 & PAFE \\
\hline Harrisius sp. & HARR & Parapsectrocladius sp. & PAPS \\
\hline Kiefferulus sp. & KIEF & Pseudosmittia sp. & PSSM \\
\hline Lauternborniella sp. & LAUT & Psectrocladius sp. & PSEC \\
\hline Paracladopelma sp. & PCLA & Subfamily Tanypodinae & \\
\hline Paratendipes sp. & PART & Ablabesmya sp. & ABLA \\
\hline Parachironomus sp. & PCHI & Alotanypus sp. & ALOT \\
\hline Phaenopsectra sp. & PHAE & Apsectrotanypus sp. & APSE \\
\hline Polypedilum sp. & POLY & Coelotanypus/Clinotanypus & COEL \\
\hline Polypedilum sp. 2 & PO16 & Djalmabatista $\mathrm{sp}$. & DJAL \\
\hline Sergentia sp. & SERG & Fittkauimyia sp. & FITT \\
\hline Stempellina sp. & STEM & Labrundina sp. & LABR \\
\hline Saetheria sp. & SAET & Larsia $\mathrm{sp}$. & LARS \\
\hline Stenochironomus sp. & STEN & Macropelopia/Apsectrotanypus & MACR \\
\hline Sublettea sp. & SUBL & Monopelopia sp. & MONO \\
\hline Xenochironomus sp. & XENO & Procladius sp. & PROC \\
\hline Tribe Tanytarsini & & Tanypodinae indet. & TAID \\
\hline Cladotanytarsus sp.1 & CLA1 & Zavrelymia sp. & ZAVR \\
\hline
\end{tabular}


TABLE 3

Diatom species $(\mathrm{n}=282)$ found in aquatic ecosystems in the Northern Neotropics. Species are ordered alphabetically within classes, orders, suborders and families. Species codes in bold $(\mathrm{n}=97)$ were included in multivariate analysis. Code indicates the species abbreviations used in figures $4 \mathrm{a}, \mathrm{b}$ and 8 . A number was designated along with the genus to identify different morphospecies. For the ecosystem studies see tables 1a,b

\begin{tabular}{|c|c|c|c|}
\hline Taxa & Code & Taxa & Code \\
\hline Class Bacillariophyceae & & Order Pennales & \\
\hline Order Centrales & & Sub-Order Araphidineae & \\
\hline Sub-Order Coscinodiscineae & & Fragilariaceae & \\
\hline Hemidiscaceae & & Campylostylus normannianus (Greville) Gerloff, & CAPS \\
\hline Actinocyclus sp. & ACTI & Natour \& Rivera 1978 & \\
\hline Melosiraceae & & Cymatosira lorenziana Grunow 1862 & CLOZ \\
\hline Hyalodiscus scoticus (Kützing) Grunow 1879 & HYSC & Fragilaria bidens Heiberg 1863 & FRBI \\
\hline Paraliaceae & & Fragilaria capucina Desmazieres emend Lange- & FCA \\
\hline Paralia sulcata (Ehrenberg) Cleve 1873 & PARA & Bertalot 1980 & \\
\hline Thalassiosiraceae & & Fragilaria capucina var. vaucheriae (Kützing) Lange- & FCAV \\
\hline Aulacoseira ambigua (Grunow) Simonsen 1979 & MA & Bertalot 1980 & \\
\hline Aulacoseira crenulata (Ehrenberg) Thwaites 1848 & AUC & Fragilaria crotonensis Kitton 1869 & FCR \\
\hline Aulacoseira distans (Ehrenberg) Simonsen 1979 & $\mathrm{MD}$ & Fragilaria famelica (Kützing) Lange-Bertalot 1980 & $\mathrm{FF}$ \\
\hline Aulacoseira granulata (Ehrenberg) Simonsen 1979 & MG & Fragilaria tenera (W. Smith) Lange-Bertalot 1980 & FT \\
\hline $\begin{array}{l}\text { Aulacoseira granulata var. angustissima (Otto Müller) } \\
\text { Simonsen } 1979\end{array}$ & MGA & $\begin{array}{l}\text { Fragilaria ulna var. goulardi (Brébisson) Lange- } \\
\text { Bertalot } 1980\end{array}$ & FRGO \\
\hline Aulacoseira granulata f. curvata (Hustedt) & MGC & Opephora marina (Gregory) Petit 1888 & OMAR \\
\hline Simonsen 1979 & & Pseudostaurosira brevistriata (Grunow in Van Heurck) & FBR \\
\hline Cyclotella atomus Hustedt 1937 & CYAT & Williams \& Round 1987 & \\
\hline Cyclotella caspia Grunow 1878 & CCAS & Staurosira construens Ehrenberg 1843 & FRAC \\
\hline Cyclotella comensis Grunow in Van Heurck 1882 & $\mathrm{CYCO}$ & $\begin{array}{l}\text { Staurosirella pinnata (Ehrenberg) } \\
\text { Williams \& Round } 1987\end{array}$ & FP \\
\hline Cyclotella cyclopuncta Håkansson \& Carter 1990 & CYCL & & $\mathrm{FHA}$ \\
\hline Cyclotella meneghiniana Kützing 1844 & CYMG & & FHA \\
\hline $\begin{array}{l}\text { Cyclotella striata (Kützing) Grunow in Cleve } \\
\& \text { Grunow } 1880\end{array}$ & CYST & Snoeijs 1992 & TABA \\
\hline Cyclotella aff. petensis & CYEN & $\begin{array}{l}\text { Tabularia fasciculata (C.A.Agradh) } \\
\text { Williams \& Round } 1986\end{array}$ & TAFA \\
\hline Cyclotella aff. striata & CAST & Ulnaria acus (Kützing) Aboal in Aboal, & SYNA \\
\hline Cyclotella sp. & CPS & Alvarez-Cobelas, Cambra \& Ector 2003 & \\
\hline Cyclotella sp. 22 & CP22 & Ulnaria delicatissima var. angustissima & FAAU \\
\hline $\begin{array}{l}\text { Discostella pseudostelligera (Hustedt) } \\
\text { Houk \& Klee } 2004\end{array}$ & $\mathrm{CCP}$ & $\begin{array}{l}\text { (Grunow in Van Heurck) Aboal in Aboal, } \\
\text { Alvarez Cobelas, Cambra \& Ector } 2003\end{array}$ & \\
\hline Discostella aff. pseudostelligera & CYAP & Ulnaria delicatissima var. angustissima & FARD \\
\hline $\begin{array}{l}\text { Discostella stelligera (Cleve et Grunow) } \\
\text { Houk \& Klee } 2004\end{array}$ & CYCS & $\begin{array}{l}\text { (Grunow in Van Heurck) Aboal in Aboal, } \\
\text { Alvarez-Cobelas, Cambra \& Ector } 2003\end{array}$ & \\
\hline Stephanodiscus hantzschii Grunow in Cleve & STHA & Ulnaria ulna (Nitzsch) Compère 2001 & SYNU \\
\hline \& Grunow 1880 & & Sub-Order Raphidineae & \\
\hline Stephanodiscus minutulus (Kützing) Cleve & STME & Eunotiaceae & \\
\hline \& Möller 1882 & & Eunotia camelus Ehrenberg 1841 & EUCM \\
\hline Stephanodiscus medius H. Håkansson 1986 & STNU & Eunotia bilunaris (Ehrenberg) Mills 1933-1935 & EUL \\
\hline Stephanodiscus parvus Stoermer \& Håkansson 1984 & STPA & Eunotia monodon Ehrenberg 1841(1843) & EUMO \\
\hline Thalassiosira sp. & THAS & Eunotia praerupta Ehrenberg 1841(1843) & EUPR \\
\hline Sub-Order Rhizosoleniineae & & Eunotia sp. & EUSP \\
\hline Biddulphiaceae & & Peronia fibula (Brébisson in Kützing) Ross 1956 & PERF \\
\hline
\end{tabular}


TABLE 3 (Continued)

Diatom species $(n=282)$ found in aquatic ecosystems in the Northern Neotropics. Species are ordered alphabetically within classes, orders, suborders and families. Species codes in bold $(\mathrm{n}=97)$ were included in multivariate analysis. Code indicates the species abbreviations used in figures $4 \mathrm{a}, \mathrm{b}$ and 8 . A number was designated along with the genus to identify different morphospecies. For the ecosystem studies see tables 1a,b

\begin{tabular}{|c|c|c|c|}
\hline Taxa & Code & Taxa & Code \\
\hline Achnanthaceae & & Brachysira sp. & BRSP \\
\hline Achnanthes minutissima var. scotica (Carter) & AMSC & Caloneis alpestris (Grunow) Cleve 1894 & CAL \\
\hline Lange-Bertalot in Lange-Bertalot \& Krammer 1989 & & Caloneis bacillum (Grunow) Cleve 1894 & $\mathrm{CAB}$ \\
\hline Achnanthidium brevipes (Agardh) Heiberg 1863 & $\mathrm{ABR}$ & Caloneis fontinalis (Grunow) Lange-Bertalot \& & CAFO \\
\hline Achnanthidium exiguum (Grunow) Czarnecki 1994 & AEX & Reichardt in Lange-Bertalot \& Metzeltin 1996 & \\
\hline Achnanthidium hungaricum Grunow 1863 & $\mathrm{AHU}$ & Capartogramma paradisiaca Novelo, & CAPA \\
\hline Achnanthidium minutissimum (Kützing) & AMI & Tavera \& Ibarra 2007 & \\
\hline Czarnecki 1994 & & Climaconeis colemaniae A.K.S.K. Prasad in Prasad, & CLIM \\
\hline Cocconeis neodiminuta Krammer 1990 & $\mathrm{CD}$ & A.K.S.K., Riddle, K.A. \& J.A. Nienow 2000 & \\
\hline Cocconeis placentula Ehrenberg 1838 & $\mathrm{CP}$ & Craticula ambigua (Ehrenberg) Mann in Round, & CRAA \\
\hline Karayevia submarina (Hustedt) Bukhtiyarova 2006 & ASUB & Crawford \& Mann 1990 & \\
\hline $\begin{array}{l}\text { Psammothidium marginulatum (Grunow) } \\
\text { Bukhtiyarova et Round } 1996\end{array}$ & AUM & $\begin{array}{l}\text { Craticula cuspidata (Kützing) Mann in Round, } \\
\text { Crawford \& Mann } 1990\end{array}$ & $\mathrm{NCU}$ \\
\hline Naviculaceae & & Craticula halophila (Grunow ex Van Heurck) & NAHA \\
\hline Amphipleura pellucida (Kützing) Kützing 1844 & APLL & Mann in Round, Crawford \& Mann 1990 & \\
\hline Amphora arenaria Donkin 1858 & AMRE & Craticula perrotettii Grunow 1867 & CRPE \\
\hline Amphora arenicola Grunow in Cleve 1895 & AMER & Cymbella mexicana (Ehrenberg) Cleve 1984 & CYMX \\
\hline Amphora copulata (Kützing) Schoeman & AMPU & Cymbella rhomboidea Boyer 1916 & CYRH \\
\hline \& Archibald 1986 & & Cymbella sp. 25 & CY25 \\
\hline Amphora cymbifera Gregory 1857 & AMCY & Diadesmis confervacea Kützing 1844 & $\mathrm{NACF}$ \\
\hline Amphora graeffeana Hendey 1973 & AMGR & Diadesmis contenta (Grunow ex Van Heurck) & $\mathrm{NCN}$ \\
\hline Amphora granulata Gregory 1857 & APTA & Mann in Round, Crawford \& Mann 1990 & \\
\hline Amphora holsaticoides Nagumo \& Kobayasi 1990 & AMHS & Encyonema densistriata Novelo, Tavera \& Ibarra 2007 & ENDE \\
\hline Amphora lybica Ehrenberg 1840 & AY & Encyonema gracile Rabenhorst 1853 & CYL \\
\hline Amphora marina W. Smith 1857 & AMAR & $\begin{array}{l}\text { Encyonema mesianum (Cholnoky) Mann in Round, } \\
\text { Crawford \& Mann } 1990\end{array}$ & CYME \\
\hline $\begin{array}{l}\text { Amphora pediculus (Kützing) } \\
\text { Grunow in Schmidt et al. } 1875\end{array}$ & AMPE & Crawford \& Mann 1990 & CYMT \\
\hline Amphora proteus Gregory 1857 & AMP & & \\
\hline Amphora securicula Peragallo \& Peragallo 1899 & AMSE & $\begin{array}{l}\text { Encyonema muelleri (Hustedt) Mann in Round, } \\
\text { Crawford \& Mann } 1990\end{array}$ & CYMU \\
\hline Anomoeoneis sphaerophora Pfitzer 1871 & ANS & Encyonema perpusillum (A. Cleve) Mann in Round, & CYPE \\
\hline Anomoeoneis sphaerophora f. costata (Kützing) & ANSC & Crawford \& Mann 1990 & \\
\hline A.-M. Schmid 1977 & & Encyonema silesiacum (Bleisch in Rabenhorst) Mann & CYML \\
\hline Anomoeoneis sphaerophora f. sculpta (Ehrenberg) & ANSS & in Round, Crawford \& Mann 1990 & \\
\hline Krammer in Krammer \& Lange-Bertalot 1985 & & Encyonema turgidum (Gregory) Grunow & CYTI \\
\hline Anomoeoneis vitrea (Grunow) Ross in Patrick \& & ANV & in Schmidt et al. 1875 & \\
\hline Reimer 1966 & & Encyonopsis angusta Krammer et Lange-Bertalot & CYAM \\
\hline Brachysira australofollis $\mathrm{H}$. Lange-Bertalot \& & BRAL & in Krammer 1997 & \\
\hline G. Moser 1994 & & Encyonopsis cesatii (Rabenhorst) Krammer 1997 & CYC \\
\hline $\begin{array}{l}\text { Brachysira hofmanniae H. Lange-Bertalot in } \\
\text { H. Lange-Bertalot \& G. Moser } 1994\end{array}$ & BROl & Encyonopsis falaisensis (Grunow) Krammer 1997 & CYF \\
\hline Brachysira neoexilis H. Lange-Bertalot & BREX & Encyonopsis microcephala (Grunow) Krammer 1997 & CYMI \\
\hline in H. Lange-Bertalot \& G. Moser 1994 & & Encyonopsis naviculacea (Grunow) Krammer 1997 & CYNV \\
\hline $\begin{array}{l}\text { Brachysira procera H. Lange-Bertalot } \\
\text { \& G. Moser } 1994\end{array}$ & BRPR & $\begin{array}{l}\text { Diploneis caffra (Giffen) A. Witkowski, } \\
\text { H. Lange-Bertalot \& D. Metzeltin } 2000\end{array}$ & DICA \\
\hline Brachysira vitrea (Grunow) R. Ross in Hartley 1986 & BRVI & Diploneis fusca (Gregory) Cleve 1894 & DFUS \\
\hline
\end{tabular}


TABLE 3 (Continued)

Diatom species $(n=282)$ found in aquatic ecosystems in the Northern Neotropics. Species are ordered alphabetically within classes, orders, suborders and families. Species codes in bold $(n=97)$ were included in multivariate analysis. Code indicates the species abbreviations used in figures $4 \mathrm{a}, \mathrm{b}$ and 8 . A number was designated along with the genus to identify different morphospecies. For the ecosystem studies see tables 1a,b

\begin{tabular}{|c|c|c|c|}
\hline Taxa & Code & Taxa & Code \\
\hline Diploneis litoralis (Donkin) Cleve 1894 & DILI & Mastogloia asperuloides Hustedt 1933 & MAAS \\
\hline \multirow{2}{*}{$\begin{array}{l}\text { Diploneis oblongella (Naegeli in Kutzing) Cleve-Euler } \\
\text { in Cleve-Euler (\& Osvald) } 1922\end{array}$} & \multirow[t]{2}{*}{ DOB } & Mastogloia braunii Grunow 1863 & MABR \\
\hline & & Mastogloia constricta Cleve 1892 & MACO \\
\hline Diploneis ovalis (Hilse in Rabenhorst) Cleve 1891 & DO & Mastogloia cyclops Voigt 1942 & MACY \\
\hline $\begin{array}{l}\text { Entomoneis paludosa (W. Smith) Reimer in Patrick } \\
\text { \& Reimer } 1975\end{array}$ & ENTO & $\begin{array}{l}\text { Mastogloia elliptica (Agardh) Cleve in } \\
\text { Schmidt et al. } 1893\end{array}$ & MASE \\
\hline \multirow{3}{*}{$\begin{array}{l}\text { Eolimna minima (Grunow in Van Heurck) } \\
\text { H. Lange-Bertalot in G. Moser, H. Lange-Bertalot } \\
\text { \& D. Metzeltin } 1998\end{array}$} & \multirow[t]{3}{*}{ NAI } & Mastogloia elliptica var. dansei (Thwaites) Cleve 1895 & MDAN \\
\hline & & Mastogloia lanceolata Thwaites in W. Smith 1856 & MLAN \\
\hline & & Mastogloia malayensis Hustedt 1942 & MALY \\
\hline $\begin{array}{l}\text { Eolimna submuralis (Hustedt) Lange-Bertalot } \\
\text { \& Kulikovskiy in Kulikovskiy et al. } 2010\end{array}$ & NAS & Mastogloia pseudoelegans Hustedt 1955 & MELP \\
\hline \multirow{2}{*}{$\begin{array}{l}\text { Eolimna aff. submuralis (Hustedt) Lange-Bertalot \& } \\
\text { Kulikovskiy in Kulikovskiy et al. } 2010\end{array}$} & \multirow{2}{*}{ NUSI } & Mastogloia pusilla Grunow 1878 & MAPP \\
\hline & & ecta Hustedt 1942 & MREC \\
\hline \multirow{2}{*}{$\begin{array}{l}\text { Fallacia pygmaea (Kützing) Stickle \& Mann in Round, } \\
\text { Crawford \& Mann } 1990\end{array}$} & \multirow{2}{*}{ NPYG } & Mastogloia smithii Thwaites in lit. ex W. Smith 1856 & MASM \\
\hline & & Mastogloia smithii var. lacustris Grunow 1878 & MASL \\
\hline Fistulifera pelliculosa (Brebisson) Lange-Bertalot 1997 & NPE & Mastogloia aff. gracilis Hustedt 1933 & MAAG \\
\hline Gomphonema affine Kützing 1844 & GAFF & Mastogloia aff. recta Hustedt 1942 & MARC \\
\hline \multirow{2}{*}{$\begin{array}{l}\text { Gomphonema amoenum Lange-Bertalot in Krammer } \\
\text { \& Lange-Bertalot } 1985\end{array}$} & \multirow[t]{2}{*}{ GOE } & Mastogloia sp. & MASP \\
\hline & & Navicula apta Hustedt 1955 & NAPT \\
\hline Gomphonema angustum Agardh 1831 & GOIM & Navicula concentrica Carter \& Ba & NCCA \\
\hline Gomphonema clevei Fricke in Schmidt et al. 1902 & $\mathrm{GC}$ & Navicula cryptotenella Lange & NRT \\
\hline Gomphonema gracile Ehrenberg 1854 & GG & & \\
\hline Gomphonema hebridense Gregory 1854 & $\mathrm{GOH}$ & Navicula eidrigiana Carter 1979 & NEDR \\
\hline Gomphonema insigne Gregory 1856 & GOIN & Vavicula flanatica Grunow 1860 & NAFN \\
\hline Gomphonema parvulum (Kützing) Kützing 1849 & GP & Navicula gregaria Donkin 1861 & NGG \\
\hline Gomphonema pseudoaugur Lange-Bertalot 1979 & GPA & Navicula hasta Pantocsek 1892 & NHAS \\
\hline \multirow{2}{*}{$\begin{array}{l}\text { Gomphonema pseudotenellum Lange-Bertalot in } \\
\text { Krammer \& Lange-Bertalot } 1985\end{array}$} & \multirow[t]{2}{*}{ GPST } & Navicula leptostriata Jørgensen 1948 & NLEP \\
\hline & & Navicula palestinae Gerloff, Natour \& Rivera 1984 & NPAE \\
\hline Gomphonema truncatum Ehrenberg 1832 & GT & Navicula perminuta Grunow in Van Heurck 1880 & NAPR \\
\hline \multirow{2}{*}{$\begin{array}{l}\text { Gomphonema vibrioides Reichardt \& } \\
\text { Lange-Bertalot } 1991\end{array}$} & \multirow[t]{2}{*}{ GOVI } & Navicula phyllepta Kützing 1844 & NAPH \\
\hline & & Navicula pseudoarvens & NPS \\
\hline \multirow{2}{*}{$\begin{array}{l}\text { Gomphonema aff. bozenae Lange-Bertalot et } \\
\text { Reichardt in Lange-Bertalot \& Metzeltin } 1996\end{array}$} & \multirow[t]{2}{*}{ GOBE } & Navicula pseudocrassirostris Hustedt 1961 & NPCO \\
\hline & & Navicula radiosa Kützing 1844 & NRA \\
\hline Gomphonema sp. & GOSP & Navicula salinarum Grunow 1880 & NRUM \\
\hline Gyrosigma baltica (Ehrenberg) Rabenhorst 1853 & GBAL & Navicula salinicola Hustedt 1939 & NASA \\
\hline Halamphora acutiuscula (Kützing) Z. Levkov 2009 & AMCU & Navicula schroeteri Meister 1932 & $\mathrm{NACH}$ \\
\hline Halamphora coffeaeformis (Agardh) Z. Levkov 2009 & AMCO & Navicula subrhynchocephala Hustedt 1935 & $\mathrm{NCHO}$ \\
\hline Halamphora montana (Krasske) Z. Levkov 2009 & AMMO & Navicula subrostellata Hustedt 1955 & NSRO \\
\hline Halamphora normanii (Rabenhorst) Z. Levkov 2009 & AMNI & Navicula subrotundata Hustedt 1945 & NSO \\
\hline Halamphora veneta (Kützing) Z. Levkov 2009 & AMVN & Navicula veneta Kützing 1844 & NAVE \\
\hline \multirow{2}{*}{$\begin{array}{l}\text { Hippodonta capitata (Ehrenberg) Lange-Bertalot, } \\
\text { Metzeltin \& Witkowski } 1996\end{array}$} & NACA & Navicula sp. & NS \\
\hline & \multirow{3}{*}{ NCHU } & Neidium ampliatum (Ehrenberg) Krammer in Kral & NEAP \\
\hline \multirow{2}{*}{$\begin{array}{l}\text { Hippodonta hungarica (Grunow) Lange-Bertalot, } \\
\text { Metzeltin \& Witkowski } 1996\end{array}$} & & \& Lange-Bertalot 1985 & \\
\hline & & Neidium iridis (Ehrenberg) Cleve 1894 & NEI \\
\hline $\begin{array}{l}\text { Luticola mutica (Kützing) Mann in Round, Crawford } \\
\& \text { Mann } 1990\end{array}$ & NAMM & Oestrupia powelli (Lewis) Heiden ex Hustedt 1935 & NPOW \\
\hline
\end{tabular}


TABLE 3 (Continued)

Diatom species $(n=282)$ found in aquatic ecosystems in the Northern Neotropics. Species are ordered alphabetically within classes, orders, suborders and families. Species codes in bold $(n=97)$ were included in multivariate analysis. Code indicates the species abbreviations used in figures $4 \mathrm{a}, \mathrm{b}$ and 8 . A number was designated along with the genus to identify different morphospecies. For the ecosystem studies see tables $1 \mathrm{a}, \mathrm{b}$

\begin{tabular}{|c|c|c|c|}
\hline Taxa & Code & Taxa & Code \\
\hline Parlibellus panduriformis John 1991 & PARP & Stauroneis schimanskii Krammer in Krammer & STSH \\
\hline Parlibellus aff. crucicula (W. Smith) A. Witkowski, & NCRU & \& Lange-Bertalot 1985 & \\
\hline H. Lange-Bertalot \& D. Metzeltin 2000 & & Stauroneis aff.schimanskii Krammer in Krammer & STAH \\
\hline Parlibellus sp. & PARL & \& Lange-Bertalot 1985 & \\
\hline Petroneis sp. & PETRO & Epithemiaceae & \\
\hline Pinnularia acrosphaeria Rabenhorst 1853 & PA & Epithemia adnata (Kützing) Brébisson 1838 & $\mathrm{EZ}$ \\
\hline Pinnularia alpina Mereschkowsky 1906 & PIAL & Epithemia turgida (Ehrenberg) Kützing 1844 & ET \\
\hline Pinnularia appendiculata (Agardh) Cleve 1895 & PIAP & Epithemia sp. & EPSP \\
\hline Pinnularia borealis Ehrenberg 1843 & PIB & Rhopalodia acuminata Krammer in Lange-Bertalot & RHAC \\
\hline Pinnularia braunii (Grunow in Van Heurck) & PBR & \& Krammer 1987 & \\
\hline Cleve 1895 & & Rhopalodia gibba (C.G. Ehrenberg 1830) & RG \\
\hline $\begin{array}{l}\text { Pinnularia cuneatiformis Krammer et Metzeltin } \\
\text { in Metzeltin \& Lange-Bertalot } 1998\end{array}$ & PICU & $\begin{array}{l}\text { O. Muller } 1895 \\
\text { Bacillariaceae }\end{array}$ & \\
\hline Pinnularia divergens $\mathrm{W}$. Smith 1853 & PIDI & Bacillaria paxillifera (O. F. Müller) Hendey 1951 & BACX \\
\hline Pinnularia interrupta W. Smith 1853 & PIIT & Denticula elegans Kützing 1844 & $\mathrm{DE}$ \\
\hline Pinnularia major (maior) (Kützing) Rabenhorst 1853 & PIMA & Denticula kuetzingii Grunow 1862 & DKU \\
\hline Pinnularia mesolepta (Ehrenberg) W. Smith 1853 & PIME & Denticula neritica Holmes \& Croll 1984 & DNER \\
\hline Pinnularia microstauron (Ehrenberg) Cleve 1891 & PIMI & Denticula subtilis Grunow 1862 & DSB \\
\hline Pinnularia subcapitata Gregory 1856 & PISU & Hantzschia amphioxys (Ehrenberg) Grunow in Cleve & HA \\
\hline Pinnularia tabellaria Ehrenberg 1843 & PITB & \& Grunow 1880 & \\
\hline $\begin{array}{l}\text { Pinnularia stomatophora (Grunow in Schmidt et al.) } \\
\text { Cleve } 1895\end{array}$ & PITO & $\begin{array}{l}\text { Hantzschia virgata (Roper) Grunow in Cleve \& } \\
\text { Grunow } 1880\end{array}$ & HAVR \\
\hline Pinnularia streptoraphe Cleve 1891 & PITR & Nitzschia acidoclinata Lange-Bertalot 1976 & NIAC \\
\hline Placoneis clementioides (Hustedt) Cox 1987 & PLCI & Nitzschia amphibia Grunow 1862 & NIAM \\
\hline Placoneis porifera (Hustedt) E.J. Cox 2003 & NPOR & Nitzschia amphibia f. frauenfeldii (Grunow) & NIFE \\
\hline Plagiotropis neovitrea Paddock 1988 & PLVI & Lange-Bertalot in Lange-Bertalot \& Krammer 1987 & \\
\hline Pleurosigma sp. & PLSP & Nitzschia amphibioides Hustedt 1942 & ND \\
\hline Rhoicosphenia abbreviata (C. Agardh) & GAB & Nitzschia bacillum Hustedt 1922 & NIBU \\
\hline Lange-Bertalot 1980 & & Nitzschia commutata Grunow in Cleve \& Grunow 1880 & NTCM \\
\hline Sellaphora densistriata $(\mathrm{H}$. Lange-Bertalot \& & SEDE & Nitzschia constricta (Kützing) Ralfs in Pritchard 1861 & NICO \\
\hline D. Metzeltin) H. Lange-Bertalot \& D. Metzeltin & & Nitzschia distans Gregory 1857 & NDIN \\
\hline in D. Metzeltin \& H. Lange-Bertalot 2002 & & Nitzschia frustulum (Kützing) Grunow in Cleve & NFRU \\
\hline Sellaphora laevissima (Kützing) D.G. Mann 1989 & NAV & \& Grunow 1880 & \\
\hline Sellaphora pupula (Kützing) Mereschkowsky 1902 & NAPP & Nitzschia frustulum var. bulnheimiana (Rabenhorst; & NBUL \\
\hline Sellaphora seminulum (Grunow) D.G. Mann 1989 & NSM & Rabenhorst) Grunow in Van Heurck 1881 & \\
\hline Sellaphora stroemii (Hustedt) H. Kobayasi in Mayama, & NSTR & Nitzschia gessneri Hustedt 1953 & NGSS \\
\hline S., Idei, M., Osada, K. \& T. Nagumo 2002 & & Nitzschia gracilis Hantzsch 1860 & NTGR \\
\hline Seminavis strigosa (Hustedt) D.G. Mann \& & AMST & Nitzschia granulata Grunow 1880 & NGRA \\
\hline A. Economou-Amilii in D.B. Danielidis & & Nitzschia grossestriata Hustedt 1955 & NIGR \\
\hline \& D.G. Mann 2003 & & Nitzschia hantzschiana Rabenhorst 1860 & NIH \\
\hline Seminavis robusta Danielidis \& D.G. Mann 2002 & SEMI & Nitzschia homburgiensis Lange-Bertalot 1978 & $\mathrm{NIHO}$ \\
\hline Seminavis pusilla (Grunow) E.J. Cox \& G. Reid 2004 & CYMP & Nitzschia inconspicua Grunow 1862 & NI \\
\hline Stauroneis anceps Ehrenberg 1843 & SA & Nitzschia lacuum Lange-Bertalot 1980 & NILA \\
\hline Stauroneis nana Hustedt 1957 & STNA & Nitzschia liebethruthii Rabenhorst 1864 & NILI \\
\hline Stauroneis phoenicenteron (Nitzsch) Ehrenberg 1843 & $\mathrm{SPH}$ & Nitzschia linearis (Agardh) W. Smith 1853 & NILN \\
\hline
\end{tabular}


TABLE 3 (Continued)

Diatom species $(n=282)$ found in aquatic ecosystems in the Northern Neotropics. Species are ordered alphabetically within classes, orders, suborders and families. Species codes in bold $(n=97)$ were included in multivariate analysis. Code indicates the species abbreviations used in figures $4 \mathrm{a}, \mathrm{b}$ and 8 . A number was designated along with the genus to identify different morphospecies. For the ecosystem studies see tables 1a,b

\begin{tabular}{|c|c|c|c|}
\hline Taxa & Code & Taxa & Code \\
\hline Nitzschia littorea Grunow in Van Heurck 1881 & NIL & Tryblionella acuminata W. Smith 1853 & NICU \\
\hline Nitzschia microcephala Grunow 1880 & NIMI & Tryblionella hungarica (Grunow) Mann in Round, & NIHU \\
\hline Nitzschia miserabilis Cholnoky 1963 & NBIS & Crawford \& Mann 1990 & \\
\hline Nitzschia nana Grunow in Van Heurck 1881 & NINA & Tryblionella levidensis W. Smith 1856 & NILE \\
\hline Nitzschia palea (Kützing) W. Smith 1856 & NPA & Tryblionella panduriformis (Gregory) Pelletan 1889 & NPAN \\
\hline $\begin{array}{l}\text { Nitzschia pararostrata (Lange-Bertalot) } \\
\text { Lange-Bertalot } 1996\end{array}$ & NIPR & $\begin{array}{l}\text { Tryblionella scalaris (Ehrenberg) P. Siver } \\
\text { \& P.B. Hamilton } 2005\end{array}$ & NSCI \\
\hline Nitzschia perminuta (Grunow in Van Heurck) & NIPE & Surirellaceae & \\
\hline M. Peragallo 1903 & & Campylodiscus echeneis Ehrenberg ex Kützing 1844 & CAEC \\
\hline Nitzschia pseudofonticola Hustedt 1942 & NIPF & Campylodiscus clypeus (Ehrenberg) Kützing 1844 & CAMPY \\
\hline Nitzschia sigma (Kützing) W. Smith 1853 & NSIG & Stenopterobia delicatissima (Lewis) Van Heurck 1896 & SUE \\
\hline Nitzschia sp. 1 & NISP1 & Surirella (Suriraya) elegans Ehrenberg 1843 & SUEL \\
\hline Nitzschia subacicularis Hustedt in Schmidt et al. 1922 & NISU & Surirella ovalis Brébisson 1838 & SUO \\
\hline Nitzschia thermaloides Hustedt 1955 & NTOI & Surirella $\mathrm{sp}$. & SUR \\
\hline Nitzschia vitrea Norman 1861 & NIVT & Surirella striatula Turpin 1816-1829 & SUTR \\
\hline
\end{tabular}

TABLE 4

Microcrustaceans found in aquatic ecosystems in the Northern Neotropics. Species are ordered alphabetically within orders and families. Species codes in bold (cladocerans $=32$; copepods $=3$; ostracodes $=17$ ) were included in multivariate analysis. Code indicates the species abbreviations used in figures 6 and 8 . For the ecosystem studies see tables 1a,b

\begin{tabular}{|c|c|c|c|}
\hline Taxa & Code & Taxa & Code \\
\hline Cladocerans $(\mathrm{n}=51)$ & & Bosminopsis deitersi Richard 1895 & $\mathrm{BDE}$ \\
\hline Order Ctenopoda & & Family Ilyocryptidae & \\
\hline Family Sididae & & Ilyocryptus spinifer Herrick 1882 & ISP \\
\hline Diaphanosoma brevireme Sars 1901 & DBR & Family Macrothricidae & \\
\hline Latonopsis australis group & LAU & Macrothrix elegans Sars 1901 & MEL \\
\hline Pseudosida ramosa Daday 1904 & PRA & Macrothrix paulensis (Sars 1900) & MPA \\
\hline Order Anomopoda & & Macrothrix cf. spinosa King 1853 & MSP \\
\hline Family Daphniidae & & Streblocerus pygmaeus Sars 1901 & SPY \\
\hline Ceriodaphnia dubia Richard 1894 & $\mathrm{CDU}$ & Family Chydoridae & \\
\hline Ceriodaphnia cf. rigaudi Richard 1894 & CRI & Alona dentifera (Sars 1901) & $\mathrm{ADE}$ \\
\hline Daphnia mendotae Birge 1918 & DME & Alona guttata group & $\mathrm{AGU}$ \\
\hline Daphnia pulicaria Forbes 1893 & DPU & Alona ossiani Sinev 1998 & AOS \\
\hline Scapholeberis armata freyi Dumont \& Pensaert 1983 & SAR & Alonella cf. excisa (Fischer, 1854) & AEX \\
\hline Simocephalus congener (Koch 1841) & $\mathrm{SCO}$ & Anthalona brandorffi (Sinev \& Hollwedel 2002) & $\mathrm{ABR}$ \\
\hline Simocephalus mixtus Sars 1903 & SMI & Anthalona verrucosa (Sars 1901) & AVE \\
\hline Simocephalus serrulatus (Koch 1841) & SSE & Camptocercus dadayi Stingelin 1900 & $\mathrm{CDA}$ \\
\hline Family Moinidae & & Chydorus brevilabris Frey 1980 & $\mathrm{CHB}$ \\
\hline Moina minuta Hansen 1899 & MMI & Chydorus eurynotus Sars 1901 & CHE \\
\hline Moinodaphnia macleayi (King 1953) & MMA & Chydorus nitidulus Sars 1901 & $\mathrm{CHN}$ \\
\hline Family Bosminidae & & Coronatella circumfimbriata (Megard 1967) & $\mathrm{CCI}$ \\
\hline Bosmina huaronensis Delachaux 1918 & BHU & Coronatella monacantha (Sars 1901) & $\mathrm{CMO}$ \\
\hline Bosmina tubicen Brehm 1953 & BTU & Dadaya macrops (Daday 1888) & DMA \\
\hline
\end{tabular}


TABLE 4 (Continued)

Microcrustaceans found in aquatic ecosystems in the Northern Neotropics. Species are ordered alphabetically within orders and families. Species codes in bold (cladocerans $=32$; copepods $=3$; ostracodes $=17$ ) were included in multivariate analysis. Code indicates the species abbreviations used in figures 6 and 8. For the ecosystem studies see tables 1a,b

\begin{tabular}{|c|c|c|c|}
\hline Taxa & Code & Taxa & Code \\
\hline Dunhevedia odontoplax Sars 1901 & DOD & Physocypria cf. denticulata (Daday 1905) & PDE \\
\hline Ephemeroporus barroisi (Richard 1894) & EBA & Physocypria globula Furtos 1933 & PGL \\
\hline Ephemeroporus hybridus (Daday 1905) & EHY & Physocypria xanabanica (Furtos 1936) & PXA \\
\hline Ephemeroporus tridentatus (Bergamin 1939) & ETR & Pseudocandona sp. & PSE \\
\hline Euryalona orientalis (Daday 1898) & EOR & Thalassocypria sp. & THA \\
\hline Graptoleberis testudinaria (Fischer 1848) & GTE & Family Cyprididae & \\
\hline Karualona muelleri (Richard 1897) & KMU & Candonocypris cf. serratomarginata (Furtos 1936) & CSE \\
\hline Kurzia longirostris Daday 1888 & KLO & Chlamydotheca colombiensis Roessler 1985 & $\mathrm{CCO}$ \\
\hline Kurzia polyspina Hudec 2000 & KPO & Cypretta cf. brevisaepta Furtos 1934 & CBR \\
\hline Leberis davidi (Richard 1895) & LDA & Cypridopsis okeechobei Furtos 1936 & $\mathrm{COK}$ \\
\hline Leydigia striata Birabén 1939 & LST & Cypridopsis vidua (Müller 1776) & CVI \\
\hline Notoalona globulosa (Daday 1898) & NGL & Eucypris sp. & EUC \\
\hline Oxyurella ciliata Bergamin 1939 & OCI & Heterocypris punctata Keyser 1975 & HPU \\
\hline Oxyuella longicaudis Birge 1910 & OLO & Potamocypris sp. & POT \\
\hline Pleuroxus quasidenticulatus Smirnov 1996 & PQU & Stenocypris major (Baird 1859) & SMA \\
\hline Pseudochydorus globosus (Baird 1843) & PSG & Strandesia intrepida Furtos 1936 & SIN \\
\hline Copepods $(n=6)$ & & Trajancypris sp. & TRA \\
\hline Class Maxillopoda & & Family Cytheridae & \\
\hline Subclass Copepoda & & Perissocytheridea cribosa (Klie 1933) & PCR \\
\hline Order Calanoida & & Family Cytherideidae & \\
\hline Family Diaptomidae & & Cyprideis sp. & CIS \\
\hline Arctodiaptomus dorsalis (Marsh 1907) & $\mathrm{ADO}$ & Family Cytheromatidae & \\
\hline Leptodiaptomus siciloides (Lilljeborg 1889) & LSI & Paracytheroma stephensoni Puri 1954 & PST \\
\hline Mastigodiaptomus nesus Bowman 1986 & MNE & Family Cytheruridae & \\
\hline Mastigodiaptomus reidae Suárez-Morales & MRE & Cytherura sandbergi Morales 1966 & CSA \\
\hline \& Elías- Gutiérrez 2000 & & Family Ilyocyprididae & \\
\hline Prionodiaptomus colombiensis (Thiébaud 1912) & PCO & Ilyocypris cf. gibba Ramdohr 1808 & IGI \\
\hline Family Pseudodiaptomidae & & Family Limnocytheridae & \\
\hline Pseudodiaptomus marshi Wright 1936 & PMA & Cytheridella ilosvayi Daday 1905 & CIL \\
\hline Ostracodes $(n=29) *$ & & Elpidium bromeliarum Müller 1880 & EBR \\
\hline Class Ostracoda & & Limnocythere floridensis Keyser 1976 & LFL \\
\hline Order Podocopida & & Limnocythere opesta Brehm 1939 & LOP \\
\hline Family Darwinulidae & & Limnocythere sp. & LIM \\
\hline Darwinula stevensoni (Brady \& Robertson 1870) & DST & Family Loxoconchidae & \\
\hline Family Candonidae & & Loxoconcha sp. & LOX \\
\hline
\end{tabular}




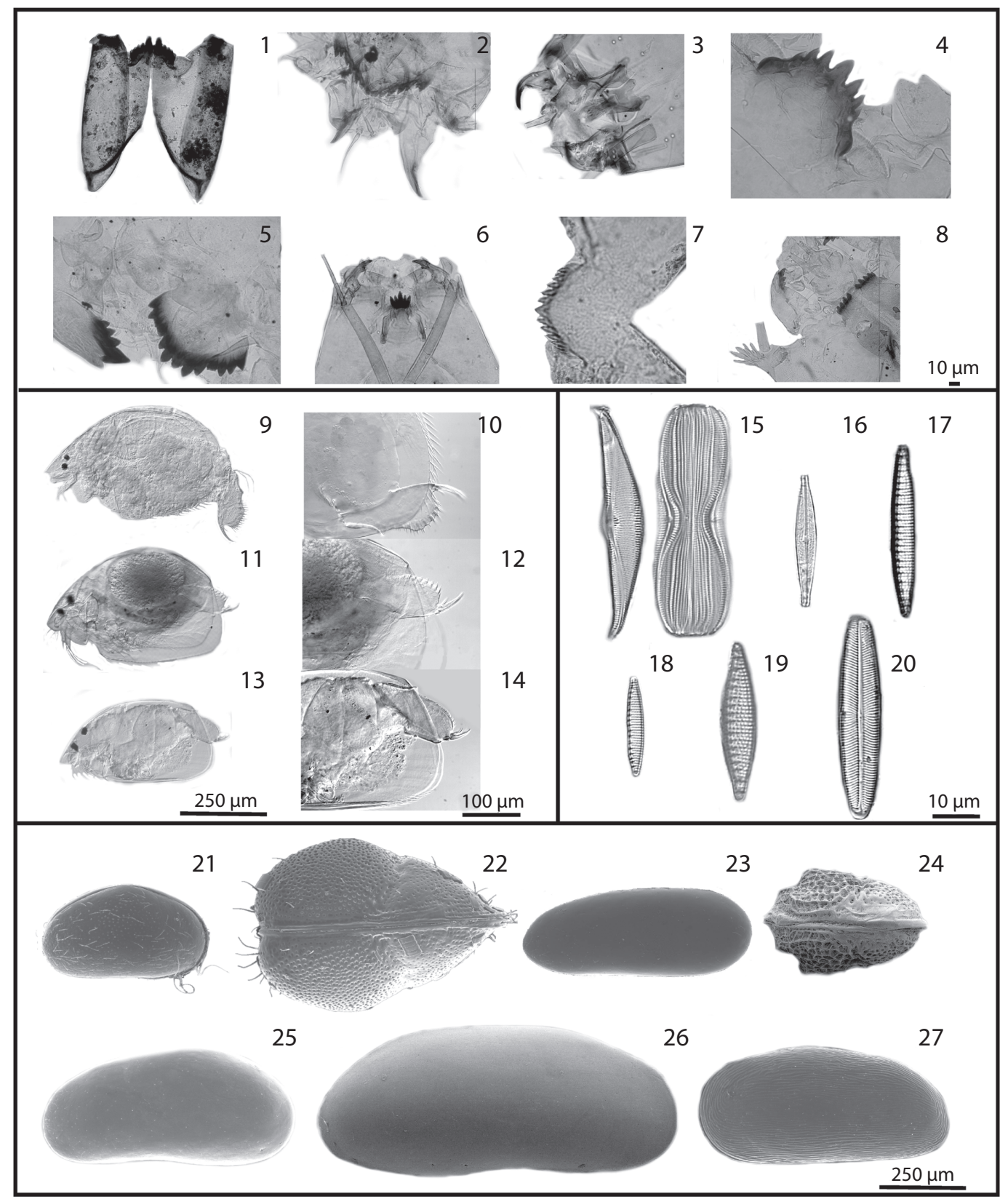

Fig. 2. Plate of selected aquatic bioindicator species. Chironomids: 1. Beardius sp., 2. Cladopelma sp., 3. Coelotanypus/ Clinotanypus, 4. Dicrotendipes sp., 5. Goeldochironomus, 6. Labrundina sp., 7. Parachironomus, 8. Stempellina sp.; Cladocerans: 9. Anthalona brandorffi $ᄋ$, 10. Postabdomen of A. brandorffi $\circ$, 11. Coronatella circumfimbriata + , 12.

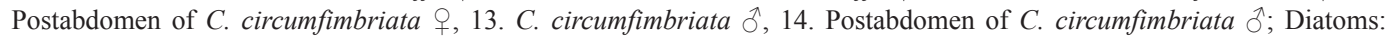
15. Amphora securicula, 16. Brachysira procera, 17. Denticula kuetzingii, 18. Nitzchia amphibia, 19. N. amphibioides, 20. Navicula palestinae; Ostracodes: 21. Cypridopsis okeechobei, 22. Cytheridella ilosvayi, 23. Darwinula stevensoni, 24. Perissocytheridea cribosa, 25. Pseudocandona sp., 26. Stenocypris major, 27. Thalassocypria sp. 


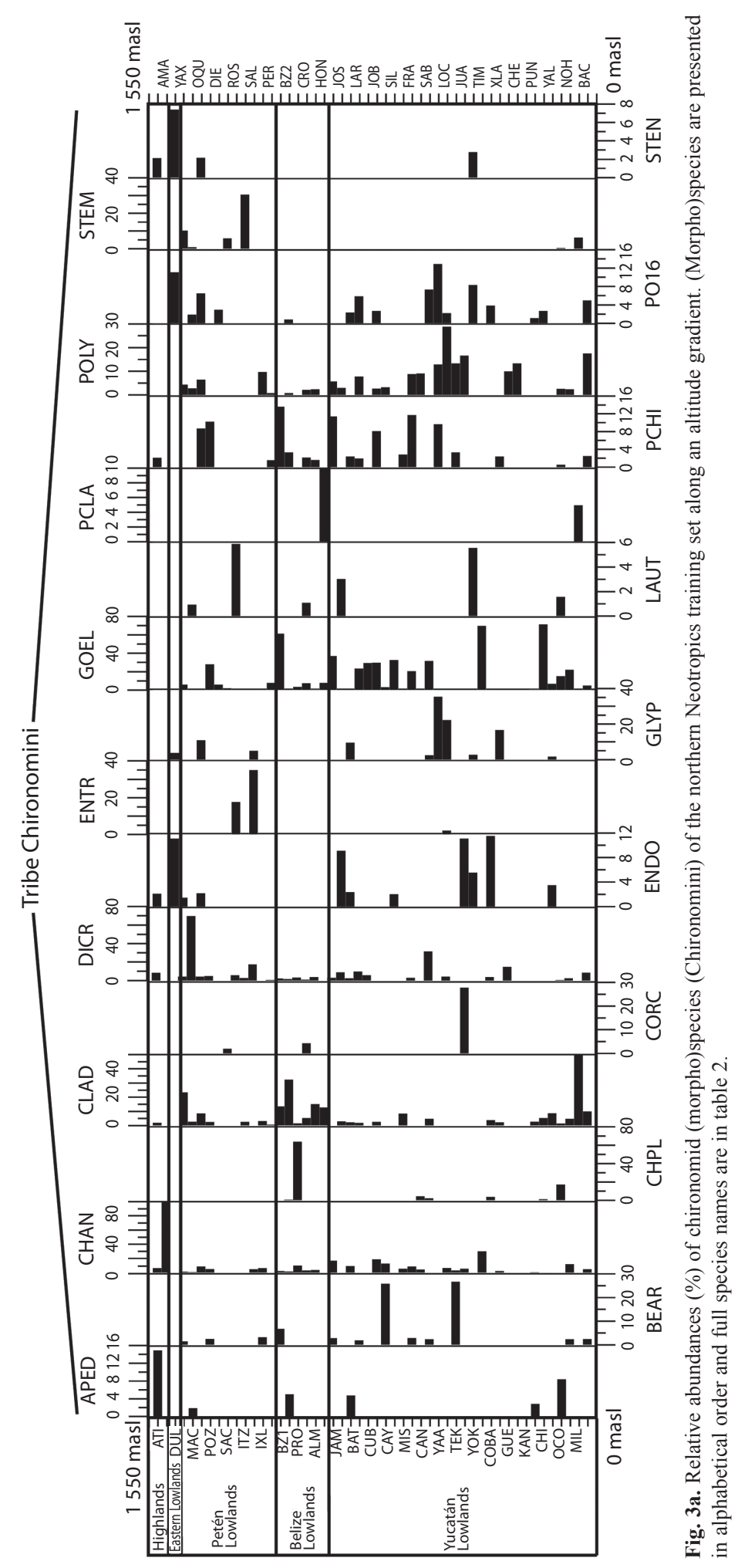




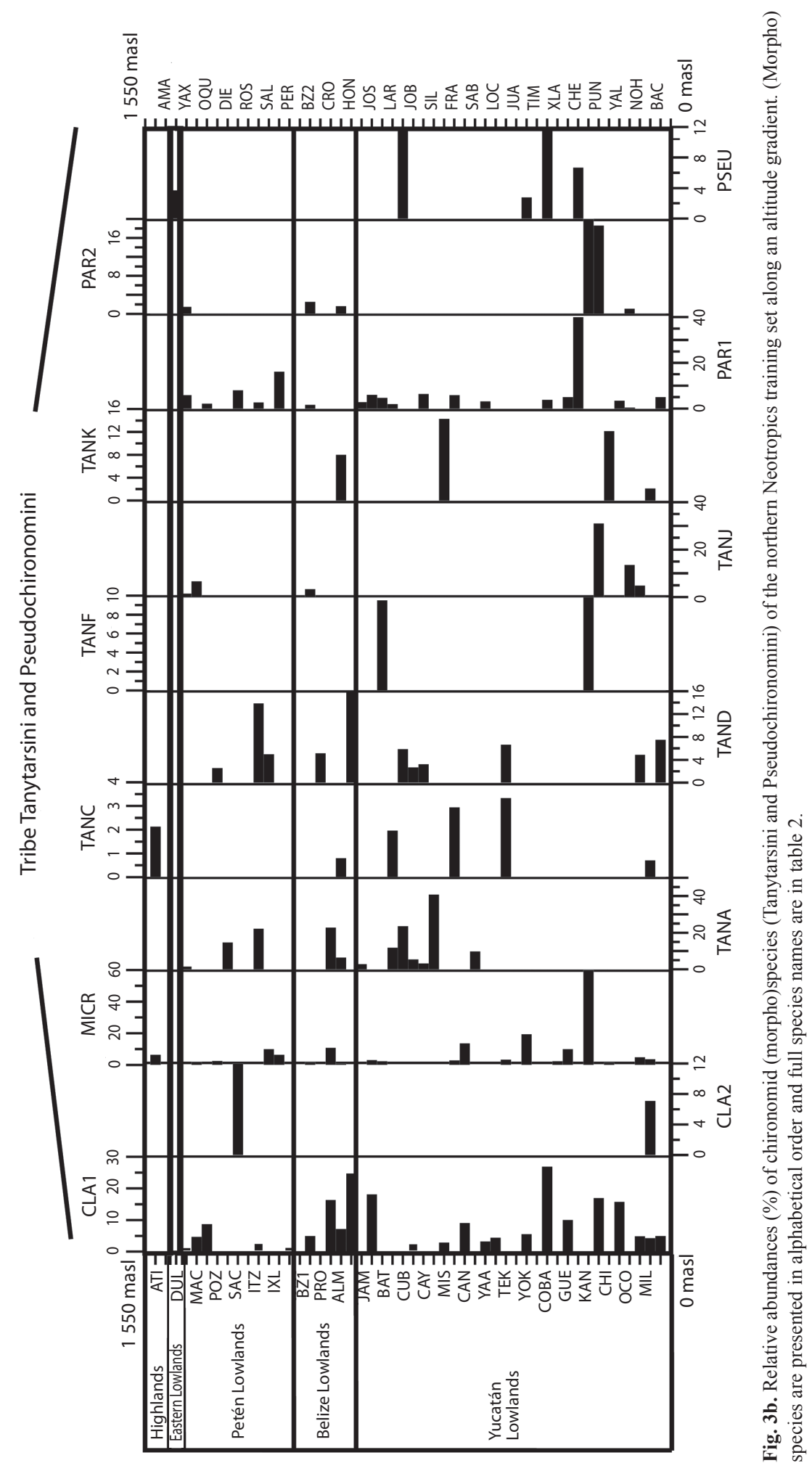




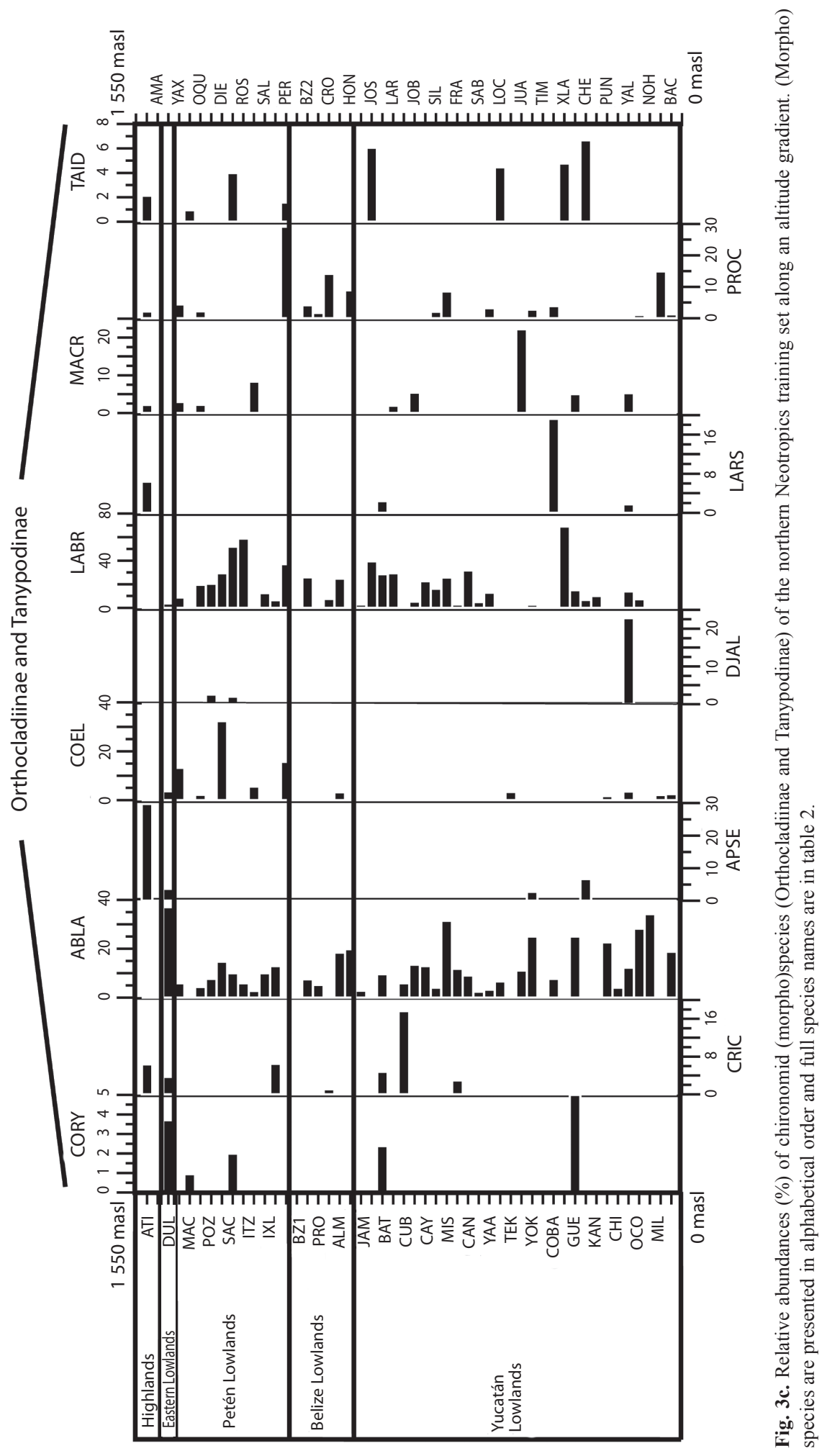


in the Belizean lowlands. Species typical of the Yucatán lowlands were Cladotanytarsus sp. 1, Goeldochironomus sp. and Polypedilum sp.1 and sp. 2.

Diatoms: Diatoms were the most abundant and diverse taxonomic group studied. Figures 4 $\mathrm{a}, \mathrm{b}$ show the most abundant diatom species ( $>2$ waterbodies). Pennate diatoms displayed the highest number of families and species. In contrast, centric diatoms were only represented by four families (Tables 3 a-e). Naviculaceae represents 162 of the 282 diatom species and were mainly distributed in lowland waterbodies on the Yucatán Peninsula. Widely distributed diatom species, i.e. those found in $>20$ waterbodies, include Brachysira procera, Cyclotella meneghiniana, Denticula kuetzingii, Encyonema densistriata, Mastogloia smithii and Nitzschia amphibia. Nitzschia amphibia and Ulnaria delicatissima var. angustissima were found in all highland lakes. Aulacoseira granulata, Fragilaria crotonensis, Ulnaria acus and Ulnaria ulna were present in three of four sampled highland lakes. The dominant species in hypereutrophic Lake Amatitlán were Cyclotella meneghiniana and Discostella aff. pseudostelligera. Fragilaria crotonensis is a species restricted to the highlands and the Eastern lowlands in Guatemala, whereas Staurosirella pinnata was only collected in Lake Izabal, in the Eastern lowlands of Guatemala. Interestingly, few waterbodies have a predominantly monospecific diatom flora, e.g. Lake Rosario (93.6\% Nitzschia amphibioides), Lake Atitlán (86.2\% Fragilaria crotonensis), Almond Hill Lagoon $81.2 \%$ N. amphibia $)$ and the pond called Belize 2 (72.6\% Encyonema densistriata).

Microcrustaceans: Cladocera were the most diverse group of microcrustacea, with 51 species belonging to seven families. Ostracodes were next, with 29 species distributed in 10 families. Calanoid copepods followed, with six species belonging to two families (Tables $4 \mathrm{a}, \mathrm{b}$ ). Figures $5 \mathrm{a}, \mathrm{b}$ and figure 6 show the relative abundances of the most widespread cladoceran, copepod and ostracode species, i.e. those present in $>2$ aquatic environments.

Cladocerans: Most collected cladoceran species belong to the order Anomopoda, family Chydoridae (Table 4 a). The greatest numbers of species were collected in lakes, ponds, and coastal waterbodies, whereas few species were collected in "cenotes" and rivers, where shells without soft parts were generally found. Assemblages in the highlands were dominated by Bosmina huaronensis, Ceriodaphnia dubia, Daphnia mendotae, Daphnia pulicaria, Moinodaphnia minuta and Simocephalus congener. Daphnia pulicaria and Simocephalus congener are restricted to highland lakes. Daphnia mendotae was the only species collected in highly productive Lake Amatitlán. Ceriodaphnia cf. rigaudi and Bosmina huaronensis displayed high relative abundance $(>35 \%)$ in the Eastern lowlands of Guatemala. Dunhevedia odontoplax was the only species restricted to the Petén and Belize lowlands, and like Ceriodaphnia dubia, was absent in the Yucatán lowlands. The greatest numbers of cladoceran species were collected in the Mexican lowlands $(n=41)$, followed by the Belizean lowlands ( $\mathrm{n}=36)$, and the Guatemalan lowlands $(\mathrm{n}=25)$. Cladoceran communities in the lowlands were dominated by Diaphanosoma brevireme, Simocephalus serrulatus, Bosmina tubicen, Ilyocryptus spinifer, Macrothrix elegans, Macrothrix cf. spinosa, Anthalona verrucosa, Chydorus brevilabris and Chydorus eurynotus. Rare cladoceran species collected in only one waterbody of the lowlands include Coronatella circumfimbriata (Loché), Dadaya macrops (Jamolún), Karualona karua (Cenote), Kurzia longirostris (Chacan-Bata) and Oxyurella ciliata (Cayucón).

Copepods: Only calanoid copepods were studied, and only six species belonging to the families Diaptomidae and Pseudodiaptomidae were identified (Table 4 b, Fig. 6). Copepod species found in highland lakes include Arctodiaptomus dorsalis, Leptodiaptomus siciloides and Prionodiaptomus colombiensis. 


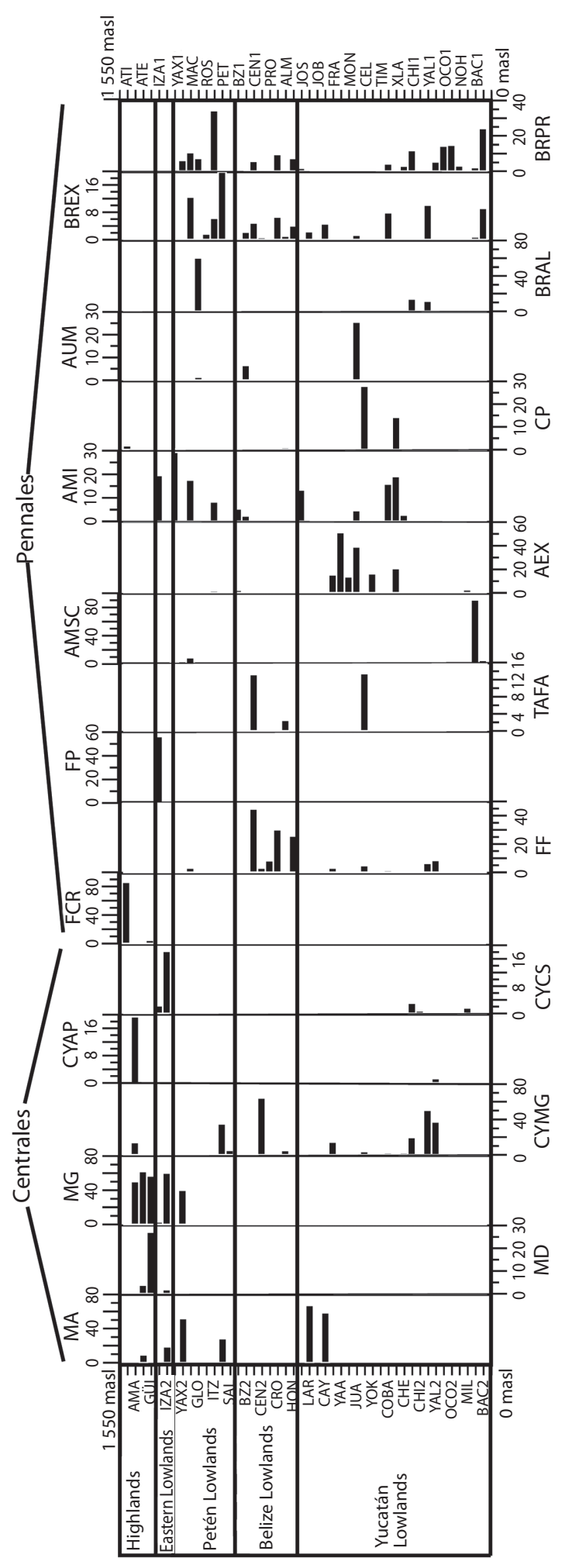

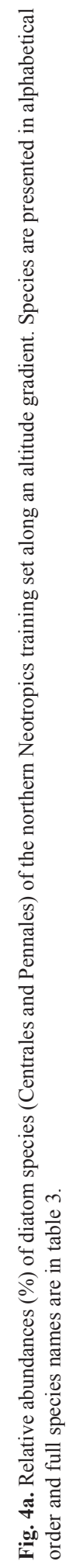




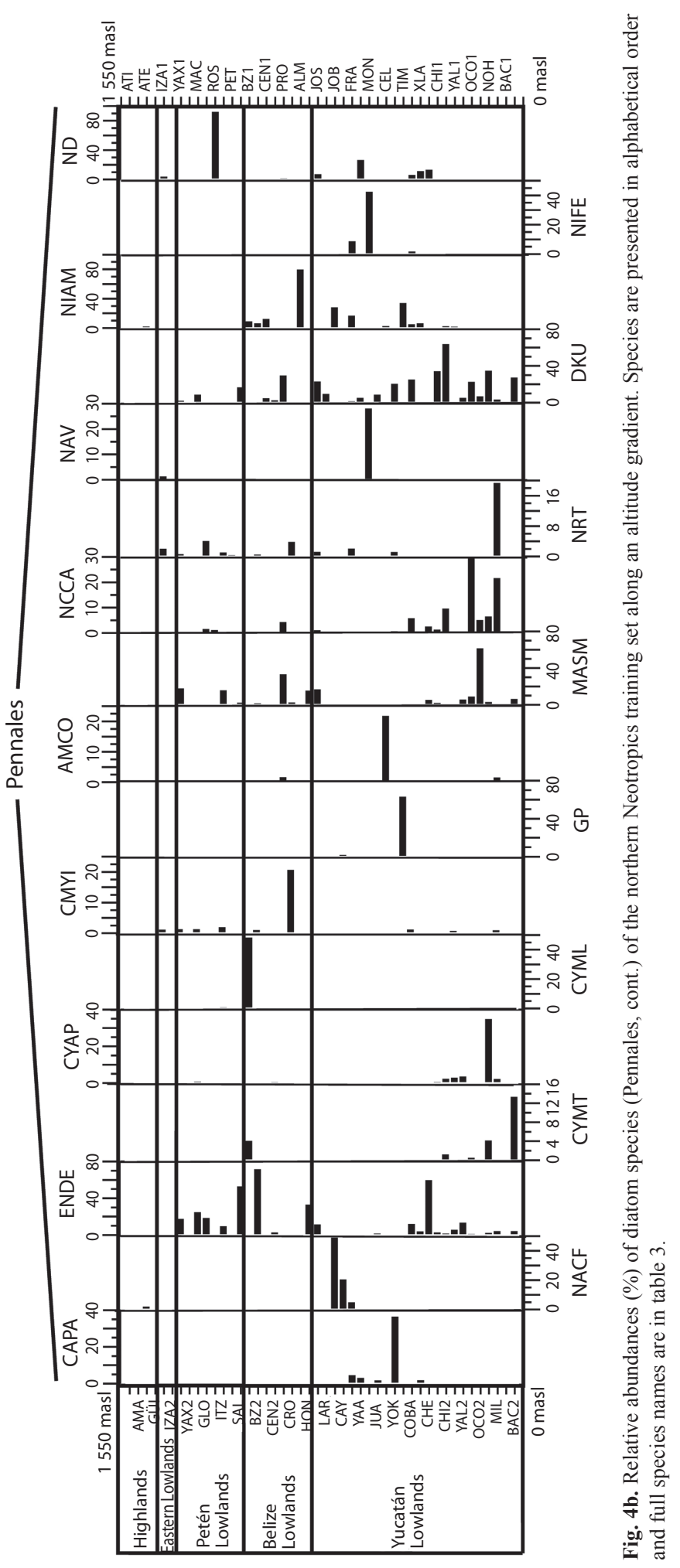




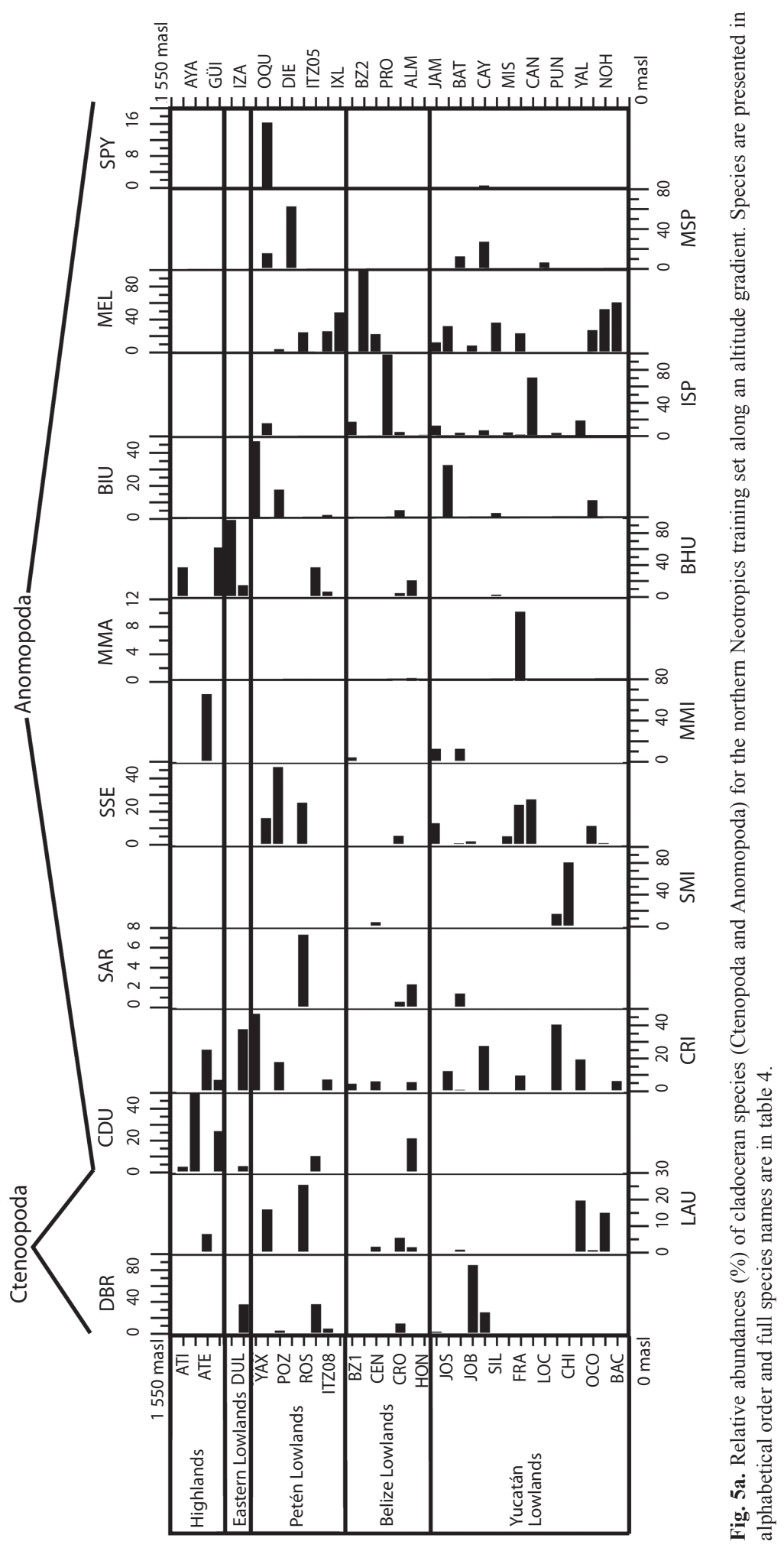




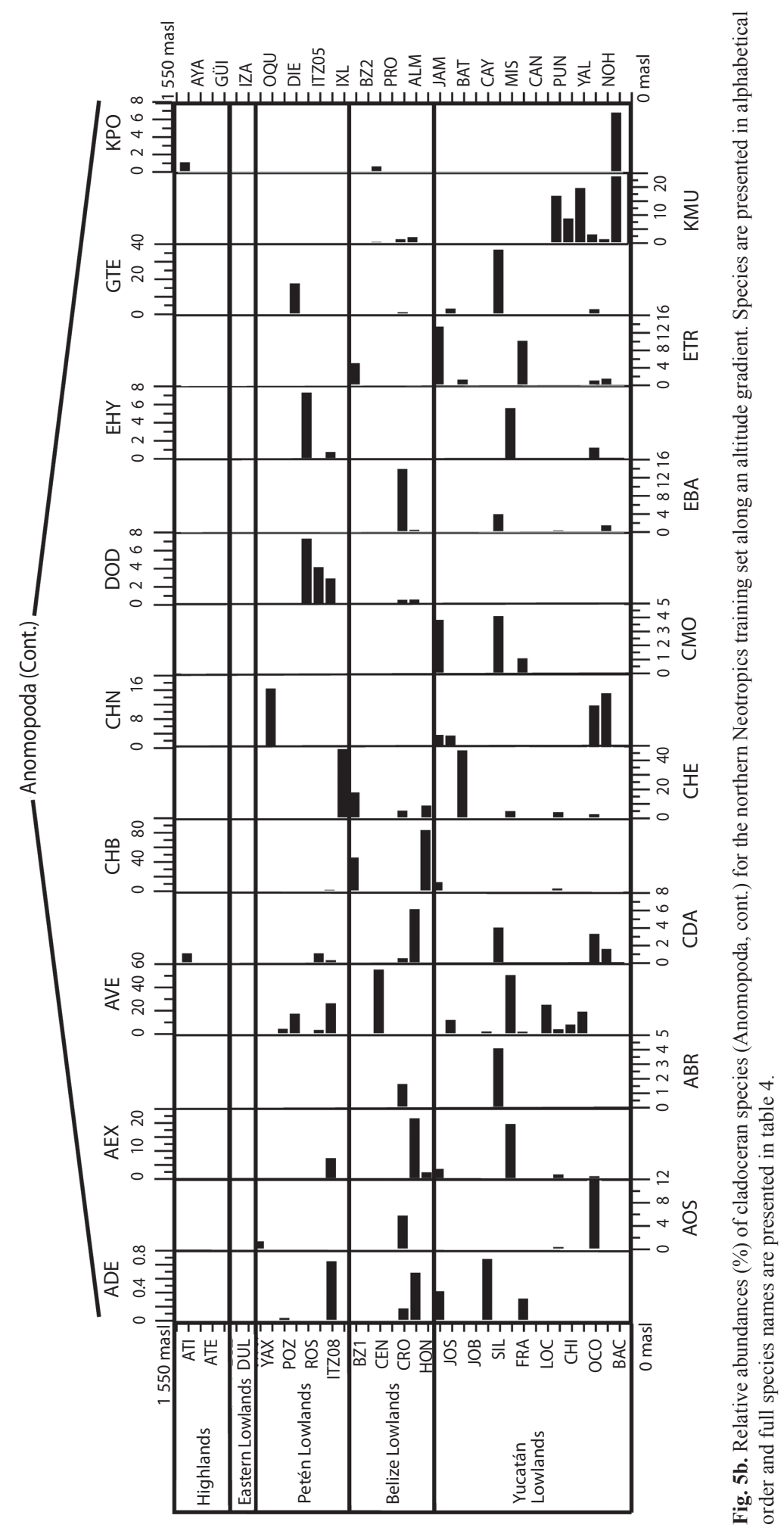




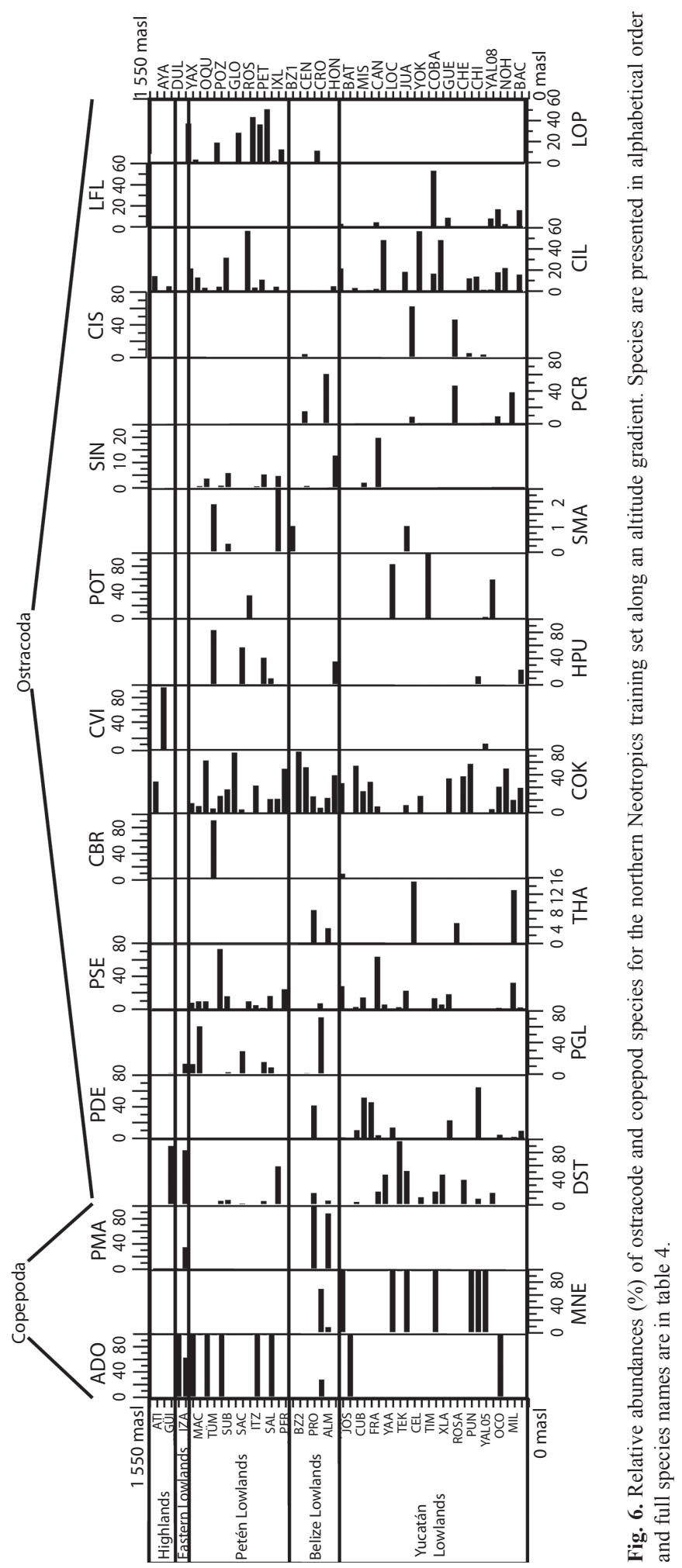


Arctodiaptomus dorsalis was the only species collected in hypereutrophic Lake Amatitlán. Leptodiaptomus siciloides and P. colombiensis are rare species that live in the highlands and were collected in the oligotrophic Laguna de Ayarza and in Lake Güija. Arctodiaptomus dorsalis was widely distributed in the lowlands, but mostly dominated aquatic ecosystems in the Petén lowlands. Mastigodiaptomus nesus is restricted to the Belize and Yucatán lowlands, whereas Pseudodiaptomus marshi inhabits the Petén and Belize lowlands. Except for Mastigodiaptomus nesus, which was found in Cenote Juarez, no calanoid copepods were collected from "cenotes" and rivers.

Ostracodes: Partial results on ostracode distribution in the Yucatán Peninsula and surrounding areas were published by Pérez et al. (2011b) and therefore only the most important results are presented here. Ostracoda was the group of microcrustaceans that displayed the highest number of families (Table $4 \mathrm{~b}$, Fig. 6). Families with highest numbers of species included Cyprididae ( $\mathrm{n}=11)$, Candonidae $(\mathrm{n}=6)$ and Limnocytheridae $(\mathrm{n}=5)$. The genera Limnocythere and Physocypria had the highest numbers of species $(\mathrm{n}=3)$. Ubiquitous species include Cypridopsis okeechobei, Cytheridella ilosvayi, Darwinula stevensoni and Pseudocandona sp. (Fig. 6). There is a clear difference between highland and lowland assemblages and between fresh and brackish water assemblages. Species typical of the highlands are Candona sp., Chlamydotheca colombiensis, Ilyocypris cf. gibba, Limnocythere sp. and Trajancypris sp. Physocypria denticulata inhabits aquatic ecosystems of the lowlands in Belize and Yucatán, whereas Physocypria globula is restricted to the Petén lowlands. Lowland rare species Cytherura sandbergi, Elpidium bromeliarum, Eucypris sp., and Physocypria xanabanica, were collected in Celestún, Río Dulce, Laguna Rosario, and in the small pond Belize 1, respectively. Cypretta brevisaepta was abundant in Lake Oquevix and in a small pond nearby.
Species richness and diversity in aquatic ecosystems: The species richness $(\mathrm{S})$ and the Shannon Wiener diversity index $(\mathrm{H})$ of diatoms, chironomids and microcrustaceans for the 63 studied aquatic ecosystems are shown in figures $7 \mathrm{a}, \mathrm{b}$. Ostracodes were collected in 59 waterbodies, chironomids in 53 and cladocerans in 46. Copepods were found in only 30 aquatic environments. Lowland waterbodies $(<450 \mathrm{~m}$. a.s.l.) displayed highest diversity values, up to $\mathrm{H}=2.6$ (diatoms), and greatest species richness, as many as 33 species (cladocerans). Lowland waterbodies Crooked Tree Lagoon, Lake Petén Itzá and Almond Hill Lagoon, followed by Lakes Yaxhá, Macanché, San José Aguilar, Cayucón, San Francisco Mateos, Cobá, Yalahau, Ocom, Nohbec, Milagros and Bacalar, yielded the highest overall species richness (up to $S=77$ ) on the Yucatán Peninsula and in surrounding areas.

Chironomids and ostracodes were present in all waterbody types (Fig. 7 a). Sampled rivers lacked diatoms and cladocerans. Copepods were scarce in rivers, "cenotes" and coastal waterbodies. The Jamolún wetland was dominated by chironomids and cladocerans. Cladocerans and ostracodes were present in all the highland lakes. Lakes Amatitlán, Gloria, Petexbatún, Celestún and Laguna Rosada displayed $\mathrm{H}$ values of 0 for chironomids, cladocerans and calanoid copepods. Ostracodes yielded H values $>0$, in TÜM, a pond near Lake Oquevix, in the Subín river and in Laguna Rosada. Cenote San Ignacio Chocholá displayed an $\mathrm{H}=0$ for all bioindicators. Sabanita yielded an $\mathrm{H}>0$ only for chironomids $(\mathrm{H}=1.8)$.

Chironomids were prominent mainly in lowland lakes. Up to 18 morphospecies were collected in Lake Yaxhá and in the pond Belize 2 , and 16 species were collected in Lakes Oquevix, Almond Hill Lagoon, Chacan-Bata, Bacalar and highland Guatemala Lake Atitlán (Fig. 7 a). Hypereutrophic Lake Amatitlán had a monospecific chironomid assemblage of Chironomus anthracinus. Dicrotendipes sp. was the only species collected in Lake Petexbatún, southern Petén. Highest diversity was reported in Lakes Oquevix $(\mathrm{H}=2.50)$ and Yaxhá 


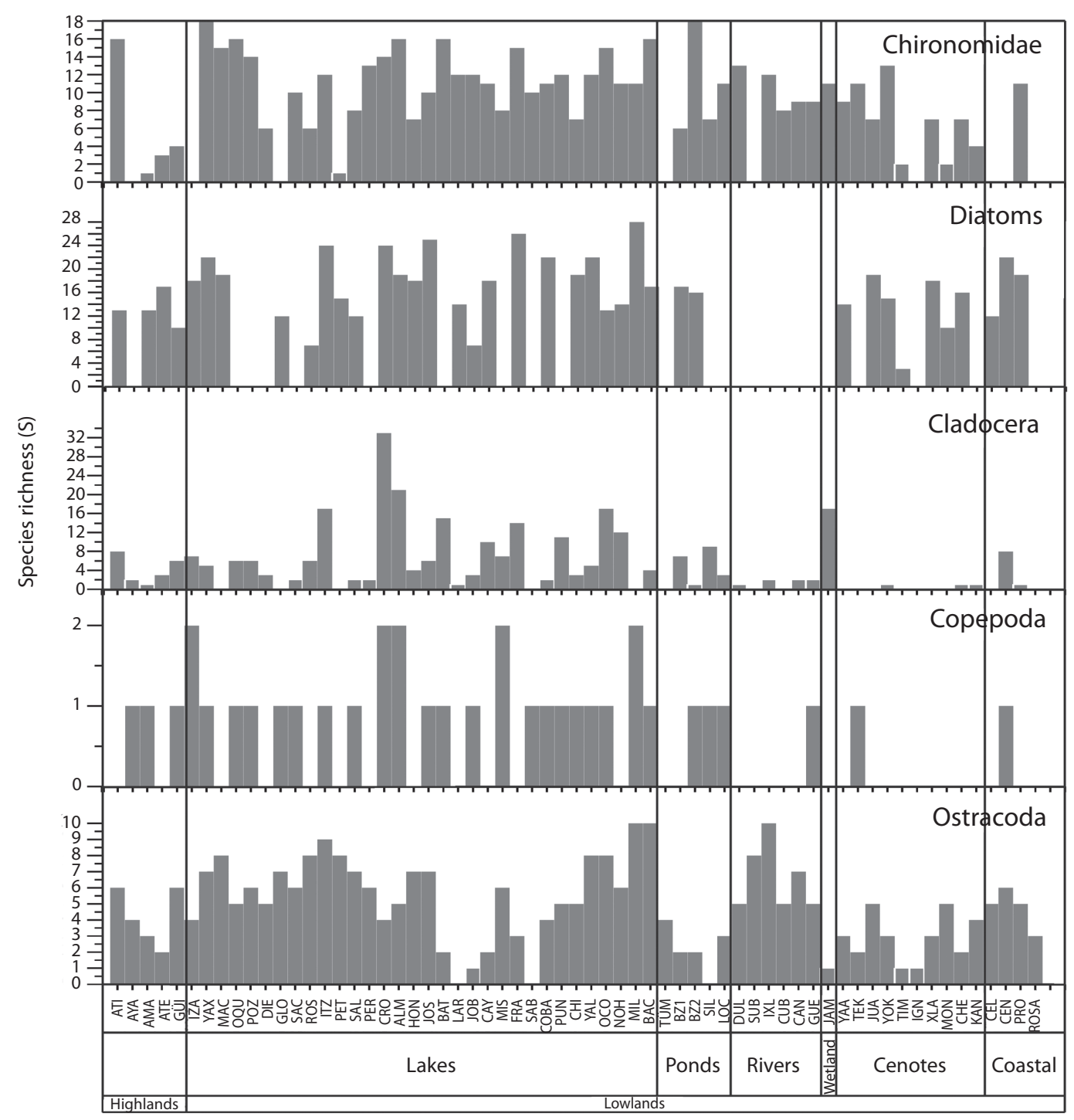

Fig. 7a. Species richness (S) of the five studied taxonomic groups (chironomids, diatoms, cladocerans, copepods and ostracodes) in aquatic ecosystems of the northern Neotropics.

$(\mathrm{H}=2.54)$. Relatively low diversities $(\mathrm{H} \leq 0.7)$ were determined in Cenotes Petén de Monos and Timul, Northern Yucatán Peninsula.

Diatoms were generally more diverse than other bioindicators in each waterbody. The number of diatom species per lake, if present, ranged from 7 to 28 . Highest numbers of species $(\mathrm{S}>20)$ were reported in Lakes Petén Itzá, Yaxhá, Cobá, Yalahau, Milagros, San
José Aguilar, San Francisco Mateos, Cenote and Crooked Tree Lagoon. Among sampled ponds, only Belize 1 and 2 possessed diatoms. In oligotrophic Crater Lake Ayarza, no diatoms were found. High diatom diversities $(\mathrm{H} \geq 2.0)$ were determined in Lakes Yaxhá, Macanché, Petén Itzá, San José Aguilar, San Francisco Mateos, Cobá, Yalahau, Milagros, Bacalar, Crooked Tree Lagoon, Cenote Xlacah and 


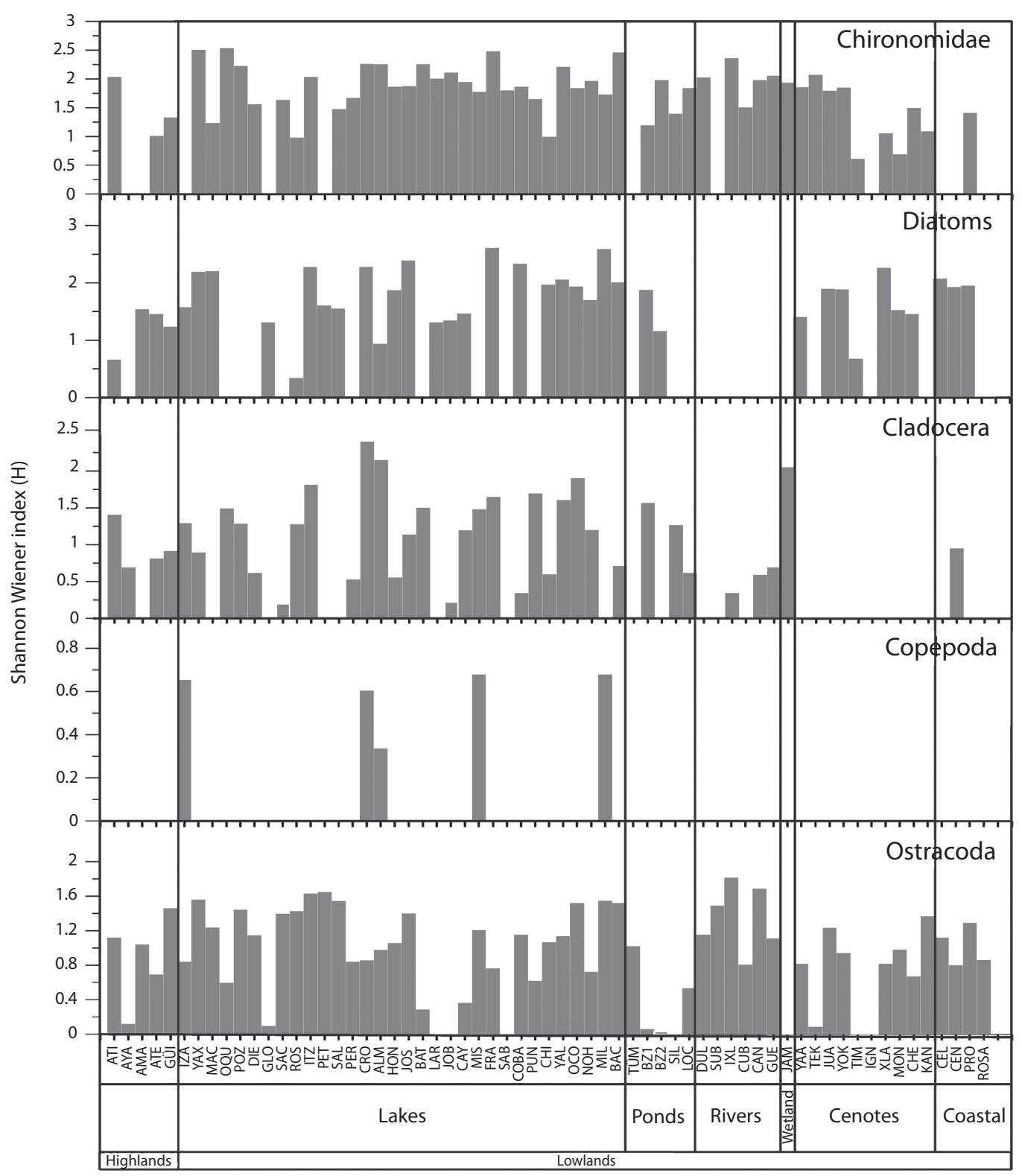

Fig. 7b. Shannon Wiener diversity index $(\mathrm{H})$ of the five taxonomic groups (chironomids, diatoms, cladocerans, copepods and ostracodes) in aquatic ecosystems of the northern Neotropics.

in coastal waterbody Celestún. In contrast, Lakes Atitlán, Rosario, Almond Hill Lagoon and Cenote Timul were characterized by low diversities $(\mathrm{H}<1.0)$.

Highest cladoceran species richness was found in Crooked Tree Lagoon (33),
Almond Hill Lagoon (21), Lakes Petén Itzá and Ocom, and in the Jamolún wetland (17). Few cladocerans were found in "cenotes" and coastal environments. The highest diversity index $(\mathrm{H}=2.4)$ was also found in Crooked Tree Lagoon. Male specimens were rare and 
reported for the cladoceran species Ceriodaphnia cf. rigauda, Diaphanosoma brevireme, Ephemeroporus barroisi, Macrothrix elegans and Macrothrix paulensis.

Highest numbers of ostracode species $(\leq 10)$ were collected in Lakes Bacalar and Milagros in Eastern Yucatán and in Ixlú River, Northern Guatemala (Fig. 7 a). The largest and deepest lake, Petén Itzá, possessed nine ostracode species. Only a few waterbodies on the Yucatán Peninsula lacked ostracodes: Chacan Lara, Sabanita and Silvituc. Ostracodes were abundant on the Yucatán Peninsula, especially in the lowlands of Petén $(S \geq 5)$. Rivers were characterized by relatively high numbers of species $(\mathrm{S}=5-10)$. Ostracodes in "cenotes" and in the Jamolún wetland were not as abundant as in other aquatic ecosystems. Ostracodes were highly diverse in rivers, and lowland lakes $(\mathrm{H} \leq 1.8)$. Ponds displayed low diversities $(\mathrm{H} \leq 0.5)$ except for a pond near Lake Oquevix (TÜM, H=1.0). Brackish waterbodies were characterized by diversity indices $\leq 1.29$.

Copepods were less abundant and diverse than chironomids, diatoms, cladocerans and ostracodes. Few calanoid copepod species $(\mathrm{S} \leq 2)$ were collected and were rarely found in rivers, "cenotes" or coastal waterbodies. Copepod diversity in the study area was $\leq 0.69$. Highest diversities were reported in Lakes Bacalar and San Francisco Mateos, followed by Lakes Izabal $(\mathrm{H}=0.65)$, Crooked Tree Lagoon $(\mathrm{H}=0.60)$ and Almond Hill Lagoon $(\mathrm{H}=0.33)$.

Calibration of bioindicators on the Yucatán Peninsula: We assessed relationships between the various studied biological groups and environmental variables. Quantitative relations between chironomids, diatoms, cladocerans and ostracodes and environmental variables were assessed using a unimodal model with 14 explanatory variables, because gradient lengths were $\geq 3$ standard deviations (SDs). The first two axes in the DCA explained 17.7\% of chironomid variability, $16.3 \%$ of diatom variability, $21.5 \%$ of cladoceran variability, and $27.8 \%$ of the ostracode species data. The sum of eigenvalues was 2.8 for chironomids,
4.3 for cladocerans, 6.1 for diatoms and 3.1 for ostracodes.

To improve the performance of the CCA model, the number of environmental variables was reduced to include only those that best explain the bioindicator distributions. Forwardselected variables displayed low inflation factors $(<5)$. Seven variables were related to chironomid $\left(\mathrm{HCO}_{3}, \delta^{13} \mathrm{C}, \mathrm{pH}\right.$, temperature, conductivity, dissolved oxygen, water depth) and diatom relative abundances (conductivity, $\delta^{18} \mathrm{O}$, dissolved oxygen, temperature, $\mathrm{pH}, \delta^{13} \mathrm{C}$, water depth), four to cladoceran (conductivity, $\mathrm{HCO}_{3}$, temperature, dissolved oxygen) and six to ostracode abundances (conductivity, $\mathrm{HCO}_{3}$, $\mathrm{Na}$, water depth, temperature, pH) (Fig. 8). For copepods, a linear model was chosen because the gradient length was only $2.15 \mathrm{SD}$ units. The first two axes in the DCA explained $72.9 \%$ of the variability in the copepod species data. The sum of eigenvalues was 1.6. Six forward-selected variables $\left(\mathrm{HCO}_{3}, \mathrm{Cl}, \mathrm{Na}\right.$, temperature, water depth, $\delta^{18} \mathrm{O}$ ) were included in the final RDA. In the final CCAs and RDA, $\mathrm{HCO}_{3}$ was the main factor controlling chironomid and copepod assemblages on the Yucatán Peninsula (Fig. 8). Diatom, cladoceran and ostracode communities are more influenced by conductivity. $\delta^{13} \mathrm{C}_{\mathrm{DIC}}$, a lake productivity proxy, and lakewater $\delta^{18} \mathrm{O}$, a proxy for changes in the balance between evaporation and precipitation and perhaps conductivity, were the second most important factors affecting chironomid and diatom distributions, respectively (Fig. 8). The final CCA for chironomids explained $4.8 \%(\lambda 1=0.14, \lambda 2=0.11)$, $6.8 \%$ for diatoms $(\lambda 1=0.42, \lambda 2=0.33), 6.4 \%$ for cladocerans $(\lambda 1=0.27, \lambda 2=0.15), 9.9 \%$ for ostracodes $(\lambda 1=0.31, \lambda 2=0.13)$ of the variability in species data, and the final RDA for copepods explained $24.9 \%(\lambda 1=0.25, \lambda 2=0.11)$ of the variability in species data (Table 5).

Chironomid species such as Stempellina sp., Goeldochironomus sp., Coelotanypus/Clinotanypus, Paratanytarsus sp. 2, Tanytarsini A and Tanytarsini $\mathrm{J}$ were positioned in the lower left quadrant of the CCA ordination biplot (Fig. 8 a). These species are typical of lowland waterbodies, especially those located on the 

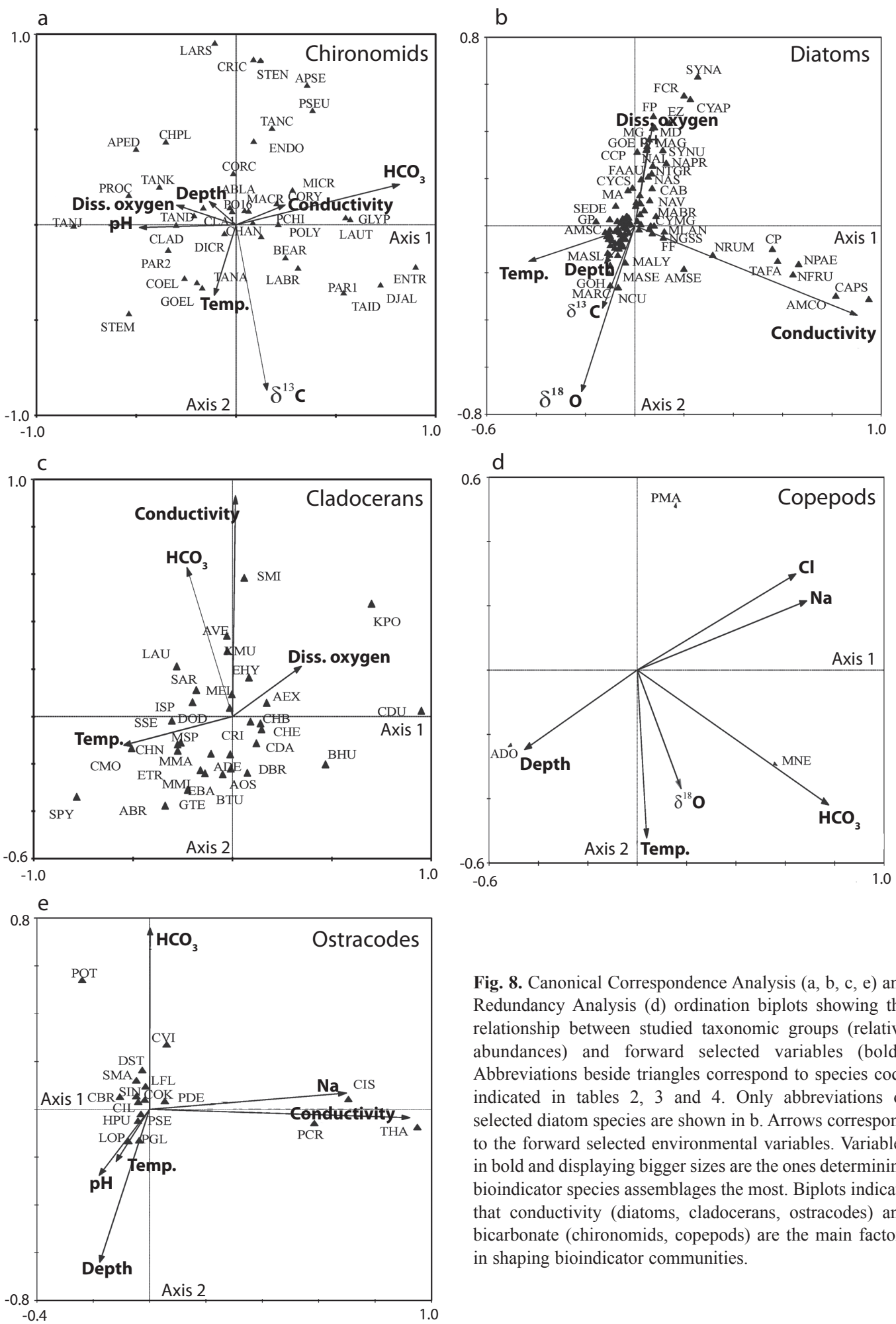

Fig. 8. Canonical Correspondence Analysis (a, b, c, e) and Redundancy Analysis (d) ordination biplots showing the relationship between studied taxonomic groups (relative abundances) and forward selected variables (bold). Abbreviations beside triangles correspond to species code indicated in tables 2, 3 and 4. Only abbreviations of selected diatom species are shown in $\mathrm{b}$. Arrows correspond to the forward selected environmental variables. Variables in bold and displaying bigger sizes are the ones determining bioindicator species assemblages the most. Biplots indicate that conductivity (diatoms, cladocerans, ostracodes) and bicarbonate (chironomids, copepods) are the main factors in shaping bioindicator communities. 
TABLE 5

Results of the Canonical Correspondence Analysis (CCA) and Redundancy Analysis (RDA) using chironomid, diatom, cladoceran, ostracode and copepod species data and forward selected variables (FSV)

\begin{tabular}{|c|c|c|c|c|c|}
\hline \multicolumn{6}{|c|}{ CCA } \\
\hline Axes & 1 & 2 & 3 & 4 & Total inertia \\
\hline \multicolumn{6}{|l|}{ Chironomids; FSV=7 } \\
\hline Eigenvalues & 0.141 & 0.110 & 0.087 & 0.080 & 2.909 \\
\hline Species-environment correlations & 0.792 & 0.781 & 0.864 & 0.738 & \\
\hline \multicolumn{6}{|l|}{ Cumulative percentage variance } \\
\hline of species data & 4.8 & 8.6 & 11.6 & 14.4 & \\
\hline of species-environment relation & 24.8 & 44.3 & 59.6 & 73.6 & \\
\hline Sum of all canonical eigenvalues & & & & & 0.567 \\
\hline \multicolumn{6}{|l|}{ Diatoms; FSV=7 } \\
\hline Eigenvalues & 0.416 & 0.329 & 0.260 & 0.184 & 6.111 \\
\hline Species-environment correlations & 0.890 & 0.947 & 0.834 & 0.831 & \\
\hline \multicolumn{6}{|l|}{ Cumulative percentage variance } \\
\hline of species data & 6.8 & 12.2 & 16.4 & 19.4 & \\
\hline of species-environment relation & 26.9 & 48.2 & 65.0 & 76.9 & \\
\hline Sum of all canonical eigenvalues & & & & & 1.545 \\
\hline \multicolumn{6}{|l|}{ Cladocerans, FSV=4 } \\
\hline Eigenvalues & 0.273 & 0.147 & 0.085 & 0.069 & 4.247 \\
\hline Species-environment correlations & 0.771 & 0.677 & 0.635 & 0.611 & \\
\hline \multicolumn{6}{|l|}{ Cumulative percentage variance } \\
\hline of species data & 6.4 & 9.9 & 11.9 & 13.5 & \\
\hline of species-environment relation & 47.5 & 73.1 & 87.9 & 100.0 & \\
\hline Sum of all canonical eigenvalues & & & & & 0.574 \\
\hline \multicolumn{6}{|l|}{ Ostracodes; FSV $=6$} \\
\hline Eigenvalues & 0.312 & 0.133 & 0.103 & 0.047 & 3.140 \\
\hline Species-environment correlations & 0.792 & 0.611 & 0.561 & 0.398 & \\
\hline \multicolumn{6}{|l|}{ Cumulative percentage variance } \\
\hline of species data & 9.9 & 14.2 & 17.4 & 18.9 & \\
\hline of species-environment relation & 49.7 & 70.9 & 87.3 & 94.8 & \\
\hline Sum of all canonical eigenvalues & & & & & 0.627 \\
\hline \multicolumn{6}{|c|}{ RDA } \\
\hline \multicolumn{6}{|l|}{ Calanoid copepodes, FSV $=4$} \\
\hline Eigenvalues & 0.249 & 0.106 & 0.039 & 0.382 & 1.000 \\
\hline Species-environment correlations & 0.637 & 0.670 & 0.510 & 0.000 & \\
\hline \multicolumn{6}{|l|}{ Cumulative percentage variance } \\
\hline of species data & 24.9 & 35.5 & 39.4 & 77.6 & \\
\hline of species-environment relation & 63.1 & 90.1 & 100.00 & 0.0 & \\
\hline Sum of all canonical eigenvalues & & & & & 0.394 \\
\hline
\end{tabular}

Eastern part of the Yucatán Peninsula, Belize, and the central and Eastern areas of the Petén Lake District. Species located in the upper left quadrant inhabit mainly lowland aquatic ecosystems, except for Apedilum sp. This species was collected at both high and low elevations, but was more abundant in highland Lake
Atitlán. Apsectrotanypus sp., Cricotopus spp., Tanytarsini C and Stenochironomus sp. inhabit highland lakes and were positioned in the right upper quadrant of the biplot. The chironomid species Glyptotendipes sp. 2 in the upper part of the right quadrant of the biplot was mainly collected in "cenotes," Lake Oquevix, Río 
Dulce and Loché pond. Chironomus anthracinus, located in the lower right quadrant, was the only species present in hypereutrophic Lake Amatitlán. Labrundina sp., Beardius sp. and Paratanytarsus sp.1 were widely distributed in the lowlands of the Yucatán Peninsula and surrounding areas.

Diatom species Halamphora coffeaeformis, Campylostylus normannianus, Nitzschia frustulum, Navicula palestinae, Tabularia fasciculata, Navicula salinarum, Amphora securicula and Cocconeis placentula are located in the lower quadrant of the biplot (Fig. 8 b) and are characteristic of lakes with high conductivities, up to $38.2 \mathrm{mS} / \mathrm{cm}$. Species characteristic of lower conductivities and most diatom species typical of fresh waters are located near the central part of the biplot.

The CCA biplot for cladocerans indicates that water conductivity influences species distribution on the Yucatán Peninsula (Fig. 8 c). Simocephalus mixtus and Karualona muelleri were positioned in the upper right and left quadrant of the CCA biplot, respectively, because they dominated lakes with high conductivities (up to $\sim 6000 \mu \mathrm{S} / \mathrm{cm}$ ) such as Cenote, Almond Hill Lagoon, Chichancanab, Punta Laguna, Yalahau, among others. Kurzia polyspina was located in the upper right quadrant because it prefers waters with dissolved oxygen concentrations between 7.3 and $8.3 \mathrm{mg} / \mathrm{L}$. Streblocerus pygmaeus, located in the lower left quadrant of the CCA biplot, is a species typical of warm lake waters $\left(>25^{\circ} \mathrm{C}\right)$ with lower conductivities $(<350 \mu \mathrm{S} / \mathrm{cm})$ such as Oquevix, Crooked Tree Lagoon and Cayucón. The dominant cladocerans in highland Lakes Atitlán, Amatitlán, Ayarza and Güija were Ceriodaphnia dubia and Bosmina huaronensis, located in the upper and lower right quadrants, respectively. Moina minuta was typical of Lake Atescatempa, Izabal, Chacan-Bata, pond Belize 1 and the Jamolún wetland. Daphnia mendotae was the only species identified in surface sediments from Lake Amatitlán.

Similar to the findings for chironomids, bicarbonate determined calanoid copepod distribution in the study area (Fig. 8 d). Few specimens were collected in the highlands of Southern Guatemala, thus all species on the biplot are typical of the lowlands. Arctodiaptomus dorsalis, in the lower left quadrant of the ordination diagram, dominated lakes with fresh waters, and was absent in "cenotes" and coastal waterbodies. Mastigodiaptomus nesus, in the lower right quadrant, is typical of $\mathrm{HCO}_{3}$ rich waters $(125-710 \mathrm{mg} / \mathrm{L})$, such as San José Aguilar, Loché, Juárez, Cobá, Punta Laguna, Chichancanab and Yalahau. Pseudodiaptomus marshi, in the upper right quadrant, is typical of lakes at low altitudes ( $<5$ m.a.s.l.), such as Lake Izabal, Guatemala and Lagoons Progreso and Almond Hill, Belize. This species was also collected in waterbodies displaying slightly higher conductivities, such as Bacalar and Lagoons Progreso and Almond Hill.

The CCA biplot for ostracodes suggests that conductivity, followed by $\mathrm{HCO}_{3}$, are the main factors controlling species distribution (Fig. 8 e). Perissocytheridea cribosa, Cyprideis sp. and Thalassocypria sp. are situated in the positive part of axis 1, indicating their preference for high-conductivity waters $(750 \mu \mathrm{S} / \mathrm{cm}$ $55.3 \mathrm{mS} / \mathrm{cm})$. Ostracode species that prefer freshwaters are situated in the center of the CCA biplot. Cypridopsis vidua, in the upper right quadrant of the CCA biplot, was more abundant in highland Lake Ayarza, Guatemala. Species tolerating the hypereutrophic water of Lake Amatitlán include Candona sp., Cypridopsis vidua and Darwinula stevensoni. Ostracodes displaying high abundances in highland and lowland lakes included Cypridopsis okeechobei, Cytheridella ilosvayi and Darwinula stevensoni. Potamocypris sp., in the upper left quadrant, was collected in Lakes Rosario, Yalahau, Loché pond and Cenote Timul, suggesting its preference for warm waters (up to $32^{\circ} \mathrm{C}$ ) and waters with $\mathrm{HCO}_{3}$ concentrations as high as $707 \mathrm{mg} / \mathrm{L}$.

\section{DISCUSSION}

Neotropical aquatic bioindicators across broad trophic and climatic gradients: We have provided a first comprehensive list of 
modern diatom (282) and chironomid (66) species for the region, along with species distributions, relative abundances in each lake, and quantitative ecological information. CCA ordination biplots, relating bioindicator species and forward selected variables, distinguish between taxa typical of highland vs. lowland lakes, brackish vs. fresh waters, alkaline vs. acidic waters, and lakes of different trophic states. Most bioindicator species live at low elevations $(<450$ m.a.s.1.), with fewer species and individuals in highland lakes. In general, diatom, cladoceran and ostracode communities are most affected by conductivity, reflecting lake water chemical composition, marine influence (Perry et al. 1995) and the N-S precipitation gradient in the Yucatán Peninsula. Species of these taxonomic groups presented characteristic faunas of fresh and brackish waters. Bicarbonate controls chironomid and copepod distribution in the study area. Concentration of bicarbonate in lake waters is an important variable in the study area because most of the studied lakes lie in karst terrain. Another related factor could be the greater abundance of edible algae in hard water lakes (Ghadouani et al. 1998). The second determinant variable for chironomid distribution was $\delta^{13} \mathrm{C}_{\mathrm{DIC}}$, an indicator of lake water productivity (McKenzie 1985), indicating the potential of some chironomid species as indicators of lake trophic state. Our results demonstrate that aquatic bioindicators on the Yucatán Peninsula are highly sensitive to changes in water column conductivity, alkalinity and trophic state.

Sánchez et al. (2002) identified 75 diatom species in "cenotes" and anchialine caves on the Eastern Yucatán Peninsula. Similar to our findings, they reported that pennate diatoms were the dominant group. Few diatoms were of marine origin. Similar results were also found in aquatic ecosystems of Costa Rica (Haberyan et al. 1997), El Salvador (Rivas Flores et al. 2010) and Nicaragua (Swain 1966). All studies indicated that Naviculaceae is a dominant family in waterbodies of the Northern Neotropics. Six species belonging to Naviculaceae, Thalassiosiraceae and Bacillariaceae were hydrochemically tolerant and displayed wide distributions: Brachysira procera, Encyonema densistriata, Mastogloia smithii, Denticula kuetzingii, Cyclotella meneghiniana and Nitzschia amphibia. Nitzschia amphibia tolerates broad trophic state and conductivity ranges. Cyclotella meneghiniana and Discostella aff. pseudostelligera dominated the hypereutrophic waters of Lake Amatitlán, Guatemala. Velez et al. (2011) used diatoms and other variables to infer environmental and cultural changes in and around this highland lake. They suggested that $C$. meneghiniana is an indicator of low lake levels, whereas $N$. amphibia indicates eutrophic waters. Highland and lowland lakes differ in their bioindicator communities, as some species are highly sensitive and restricted to specific areas. Fragilaria crotonensis is a species typical of the highlands and Eastern lowlands in Guatemala. Fragilaria species indicate oligotrophic to mesotrophic conditions (Castellanos \& Dix 2009). This species dominated $(86.2 \%)$ in Lake Atitlán, a lake that experienced extensive cyanobacteria (Lyngbya hieronymusii/birgei/robusta) blooms in October 2009 (Rejmánková et al. 2011). When we visited Lake Atitlán in March 2008, the lake still displayed oligo- to mesotrophic conditions, indicated by the dominance of $F$. crotonensis, shortly before the first cyanobacteria bloom, which occurred in December 2008.

Vinogradova \& Riss (2007) reported 84 chironomid taxa, mainly morphospecies, from 18 lakes on the Yucatán Peninsula. In their study, the dominant chironomid species were Cladopelma lateralis and species belonging to the genus Tanytarsus. Our dataset included a larger number of aquatic ecosystems $(n=63)$, however results from both studies are similar. Few chironomid taxa are restricted to specific areas. Rather, the dipterans seem to tolerate a broad range of environmental conditions. Chironomus anthracinus displayed high relative abundance in most sampled waterbodies and the larvae have been to shown to be among the dominant food items of fish (Armitage et al. 1995). This species was very abundant in many of our surface sediment samples. It 
tolerates eutrophic waters (Porinchu \& MacDonald 2003), which characterize many lowland and some highland lakes in the study area. For instance, C. anthracinus was the only dipteran species collected in hypereutrophic Lake Amatitlán, Guatemala. For decades, this highly productive lake has received wastewater, delivered by its main inflow river, the Río Villalobos. This species was also collected in Cenote Timul, which displayed high $\delta^{13} \mathrm{C}_{\text {DIC }}$ values of $+13.6 \%$ (Pérez et al. 2011a). These results illustrate that $C$. anthracinus can be used as an indicator of highly productive waters in the Northern Neotropics. A larger number of species $(n=51)$ inhabit the lowlands. Fewer species were identified in the highlands $(n=15)$, suggesting that chironomids are very abundant in low-elevation neotropical regions, similar to findings in Africa (Eggermont et al. 2010), where 81 chironomid taxa were collected across an altitude gradient (489-4 575 m.a.s.1.) and in Brazil (de Oliveira Roque \& Trivinho-Strixino 2007), where 191 morphospecies were collected.

Cladocerans dominated the microcrustacean communities in the study area. Fifty-one species were collected in the waterbodies and the greatest number of species belonged to the family Chydoridae. Many species of Chydoridae have great value as water-quality indicators because they are highly sensitive to changes in lake trophic state (de Eyto et al. 2002). Cladocerans and copepods are the two taxonomic groups most studied on the Yucatán Peninsula and in surrounding areas (Elías-Gutiérrez et al. 2008). Mexico has been actively involved in studying the systematics of Cladocera (ElíasGutiérrez et al. 2006). Therefore, identification of collected cladocerans and copepods to species level was possible. Elías-Gutiérrez (2006) reported a total of 162 cladoceran species for two regions of Mexico (Morelos and southeast Mexico), four being endemic species of Southeast Mexico. Some of the cladoceran species we collected are widely distributed in the Northern Neotropics and South America. These include Diaphanosoma brevireme, Pseudosida ramosa, Macrothrix spinosa, M. elegans, Chydorus nitidilus, Ephemeroporus tridentatus, Alona ossiani, Oxyurella ciliata and O. longicaudis (Elías-Gutiérrez 2006). One interesting finding of our study was the presence of Anthalona brandorffi, described as Alona brandorffi (Sinev \& Hollwedel 2002) in the waterbodies Crooked Tree Lagoon, Silvituc Lagoon and Loché, because this species was found for the first time in Boa Vista, Brazil, and its distribution in the Northern Neotropics was unknown. Cladocerans were not as abundant in highland lakes of Guatemala. Laguna de Ayarza and Lake Atitlán still display oligo-mesotrophic conditions. Macrophytes, the typical habitat of cladocerans, are scarce in these lakes. In contrast, Lake Amatitlán is hypereutrophic, and only a single cladoceran species, Daphnia mendotae, was collected in such extreme conditions. Species restricted to the highlands include $D$. pulicaria and $S$. congener, even though they apparently have wide distributions (Cerny \& Hebert 1993, Illyová \& Némethová 2005, Marrone et al. 2005). For instance, $D$. pulicaria seems to prefer cool waters, typical of highland Lake Atitlán $\left(\leq 21.8^{\circ} \mathrm{C}\right)$. Occupying cooler, deep waters may be a strategy to reduce risk of predation (Stich \& Maier 2007). Species restricted to aquatic ecosystems of Guatemala and Belize include: Dunhevedia odontoplax and Ceriodaphnia dubia. Dunhevedia odontoplax has been collected in Morelos, Veracruz (Elías-Gutiérrez 2006). Mainly cladoceran carapaces were collected in "cenotes" and rivers, but there were few live specimens. Most "cenotes" we sampled lacked aquatic vegetation, the main habitat of most cladoceran species. Distribution of zooplankton in rivers is very heterogenous (Vadadi-Fülöp 2009) and we might simply have collected samples from sites where densities were low. Scarcity of cladocerans in rivers, however, is common because they are not as well adapted to lotic aquatic environments as ostracodes and chironomid larvae. Another explanation for the low species richness and numbers could be that adults of some species are more typical of the rainy season, and we collected surface sediments in the dry 
season. Future sampling should be conducted across the seasons.

Only six calanoid copepod species were collected from the sampled waterbodies. Recent studies in Mexico (Elías-Gutiérrez et al. 2008, Brandorff 2012) report up to 100 freshwater copepod species, of which 20 species belong to the order Calanoida. SuárezMorales \& Reid (2003) suggest that the fauna of the Yucatán Peninsula has affinities with Cuba and the insular Caribbean, and differs from that of Central Mexico, which is closer to the fauna of upper Central America. Prionodiaptomus colombiensis mainly inhabits altitudes from 10 to 100 m.a.s.l and it has been previously reported in Tabasco, Mexico while Leptodiaptomus siciloides is widely distributed in Mexico (Elías-Gutiérrez et al. 2008). Arctodiaptomus dorsalis tolerates a broad range of environmental conditions and was collected in waterbodies with different origins and trophic states. For instance, it inhabits hypereutrophic volcanic Lake Amatitlán in the highlands, and meso-oligotrophic, karst Lake Petén Itzá in the lowlands. According to Suárez-Morales (2003), this nearctic species is the most widespread diaptomid in the Yucatán Peninsula and has also been collected in Southeastern USA, central and Eastern Mexico, Central America and the Caribbean islands. Dispersal of this species is relatively recent (post-Pliocene) and it colonized the Yucatán Peninsula during past marine regressions, during times of emergence of areas on the peninsula. This could explain why this species is now highly tolerant and widely distributed.

Of the six copepod species found, M. reidae is endemic to Campeche (Elías-Gutiérrez et al. 2008, Suárez-Morales \& Elías-Gutiérrez 2000) and Northern Guatemala (this study). All species belonging to the genus Mastigodiaptomus found in the Yucatán Peninsula are neotropical. Suárez-Morales (2003) report another endemic species for the area, Mastigodiaptomus maya. Unfortunately, we did not collect this species, but it seems to coexist with $M$. reidae in Chicaná pond, near the Biosphere Reserve of Calakmul, Yucatán Peninsula, and probably speciated for ecological reasons. Our results indicate that $M$. nesus inhabits waterbodies in Belize, Campeche, Quintana Roo and Yucatán, as reported by Elías-Gutiérrez et al. (2008). The present distribution of this taxon could be a remnant of the original Mastigodiaptomus fauna in the Yucatán Peninsula and may reflect recent, post-Pliocene dispersal and Holocene climatic fluctuations (Suárez-Morales 2003). We identified $P$. marsh i in aquatic ecosystems in the lowlands of Belize (Lagoons Progreso and Almond Hill) in the Eastern lowlands of Guatemala (Lake Izabal) and Southern Yucatán (Bacalar). Pseudodiaptomid copepods mainly inhabit marine and brackish water environments, although recent studies (Suárez-Morales 2003) suggest that $P$. marshi is a species that is starting to colonize freshwater environments. Canonical Correspondence Analysis indicated that the ions sodium and chloride affect the distribution of this species. But the fact that we collected this copepod species in freshwater Lake Izabal supports the idea that is starting to colonize freshwater environments. Lake Izabal is connected with the Caribbean Sea via the Río Dulce and El Golfete. Similar to cladocerans, few copepods were found in rivers, probably because they are not well adapted to inhabit running waters, avoiding such environments and preferring the littoral zones of lakes (Casanova \& Henry 2004).

Effects of altitude and related variables precipitation and trophic state, on ostracode species distribution and assemblage composition in the study area are clear. The taxonomy, ecology and distribution of non-marine ostracodes from the Northern Neotropics (Mexico, Guatemala and Belize) was investigated by Pérez et al. (2010b, 2010c, 2011b), Darwinula stevensoni has a worldwide distribution and Cytheridella ilosvayi is abundant throughout the entire continental Neotropics. Cypridopsis okeechobei displays a narrower distribution, extending from the United States to the Petén Lake District, Northern Guatemala. Pseudocandona sp. was also abundant and we suggest this species is endemic to the Yucatán Peninsula, but further taxonomic and molecular 
analysis is needed to test this assertion. We were unable to identify some ostracodes collected in the highlands to species level. They may be endemic to the region or be distributed throughout higher-elevation areas of Central America and Mexico that have not been studied yet. These species include Candona sp., Limnocythere sp. and Trajancypris sp. A conductivity gradient is well marked on the Yucatán Peninsula. Ostracodes were mainly typical of freshwaters, but some, like Cyprideis sp., Loxoconcha sp., Paracytheroma stephensoni, Perissocytheridea cribosa and Thalassocypria sp. were typical of waterbodies with high conductivities, up to $55.3 \mathrm{mS} / \mathrm{cm}$. Cypretta brevisaepta had been reported only from Southern Florida and the West Indies, but we found it in Lakes Oquevix, Macanché, and a pond near Lake Oquevix in Petén, Guatemala and in San José Aguilar, Mexico.

A broad trophic state gradient characterizes the study area, ranging from hypereutrophic Lake Amatitlán to oligotrophic Laguna Ayarza. Hypereutrophic Lake Amatitlán displayed species characteristic of highly productive waters, including Chironomus anthracinus, Discostella aff. pseudostelligera, Daphnia mendotae, Candona sp., Cypridopsis vidua and Darwinula stevensoni. Our results demonstrate that few zooplankton and zoobenthos species inhabit higher elevations ( $>450$ m.a.s.l.) in Guatemala. Cladocerans, copepods and ostracodes were more diverse and abundant in lowland aquatic ecosystems, suggesting that environmental conditions in those waterbodies are optimal for zooplankton and zoobenthos development and reproduction. The Yucatán Peninsula and surrounding areas (Guatemala and Belize) are rich in aquatic ecosystems, therefore it will be important to collect samples from additional sites to expand our training set. Aquatic bioindicators should also be collected at different seasons to provide information on species life cycles. Despite the utility of the collected data, additional sampling campaigns in aquatic ecosystems throughout Mexico, Guatemala, Belize and Central America are required. We also recommend return visits to previously studied ecosystems to capture seasonal variability. Central Mexico is rich in aquatic ecosystems and there have been few studies on bioindicators in that region. We are developing a calibration dataset for central Mexico that will provide new autecological information for bioindicators that will expand our original Yucatan training set.

Importance of species richness and diversity of bioindicators in neotropical aquatic ecosystems: Diversity and species richness data from waterbodies provide information on modern environmental conditions, e.g., trophic state, anthropogenic impact and urban development and the degree of degradation. Our findings provide information for identifying conservation hotspots on the Yucatán Peninsula, Guatemala and Belize. Highest species diversities were reported at lower elevations $(<450 \mathrm{~m}$.a.s.1.). The highest number of species and diversities per waterbody were usually reported for lowland lakes, where precipitation is high, up to $3050 \mathrm{~mm} / \mathrm{y}$. Crooked Tree Lagoon, Belize displayed the highest diversity ( $\mathrm{H} \leq 2.4$, diatoms). The lagoon is protected and recognized as a wetland of international importance under the Ramsar Convention of Wetlands (http://www.ramsar.wetlands.org). Lakes Bacalar and Chichancanab, on the Yucatán Peninsula, were declared protected areas in April 2011 (SIPSE 2011). Most aquatic ecosystems in the study area, however, lack such environmental protection. Government agencies, universities and NGOs should collaborate to guarantee that aquatic ecosystems in the region are protected. Highland lakes, despite their lower diversities, deserve special attention because they often possess rare or unidentified taxa and may be home to new or endemic species. Chironomids and ostracodes were highly diverse $(\mathrm{H} \leq 2.54)$ in sampled aquatic ecosystems. Lakes Chacan Lara, Sabanita and Silvituc were small waterbodies that lacked ostracodes, probably due to low lake water conductivities $(\leq 183 \mu \mathrm{S} / \mathrm{cm})$. Diatoms, cladocerans and copepods were scarce or lacking in rivers, "cenotes" and coastal waterbodies. Further sampling 
campaigns are needed to corroborate these observations and improve on methods for collection of bioindicators that were present in low abundances. Rivers deserve special attention because they frequently receive domestic and industrial waste, affecting species distributions and diversity.

This study on the waterbodies on and around the Yucatán Peninsula found that microcrustacea, insect larvae and diatoms in neotropical lakes are abundant, diverse and highly sensitive to environmental variables. Such organisms therefore have great potential as modern and late Quaternary bioindicators. This investigation generated the first training sets for chironomids, diatoms, ostracodes, cladocerans and copepods in the region and is a pre-requisite for future quantitative paleolimnological reconstruction of late Quaternary environments in the Northern Neotropics. Our results highlight the exceptional potential of the studied taxonomic groups as bioindicators of climate and trophic state.

Analysis of the distribution and ecological preferences of the five studied groups (diatoms, chironomids, cladocerans, copepods and ostracodes) generated new information that is required to make better use of these aquatic bioindicators in the neotropical region. Clear differences emerged in the chemistry and biology of highland versus lowland water bodies. Volcanic highland lakes display origin and water chemical composition different from those of karst lowland lakes. Biodiversity in the highlands is lower than in the lowlands. The highland aquatic fauna is dominated by chironomids Apsectrotanypus sp., Cricotopus spp., Tanytarsini C, Stenochironomus sp., diatoms Ulnaria acus, cladocerans Ceriodaphnia dubia and Bosmina huaronensis, ostracodes Candona sp., Chlamydotheca colombiensis, Cypridopsis vidua, Cytheridella ilosvayi, Darwinula stevensoni, Limnocythere sp., Physocypria globula, Stenocypris major and Trajancypris sp, cladocerans $D$. pulicaria and $S$. congener and the copepods Leptodiaptomus siciloides and Prionodiaptomus colombiensis.
This study covered a wide range of trophic state, which allowed us to differentiate between species that are tolerant and intolerant of highly eutrophic waters. Bioindicator species inhabiting highly productive waters and tolerating extreme conditions include Chironomus anthracinus, Cyclotella meneghiniana, Discostella aff. pseudostelligera, Daphnia mendotae, Arctodiaptomus dorsalis, Candona sp., Cypridopsis vidua and Darwinula stevensoni. A broad conductivity gradient also characterizes the Yucatán Peninsula and surrounding areas. Most collected species inhabit freshwaters, but a few tolerate high conductivities, making them potential indicators of such conditions. They include diatoms such as Halamphora coffeaeformis, Campylostylus normannianus, Nitzschia frustulum, Navicula palestinae, Tabularia fasciculata, Navicula salinarum, Amphora securicula, and Cocconeis placentula, cladocerans Simocephalus mixtus and Karualona muelleri, the copepod species $P$. marshi, as well as ostracodes Cyprideis sp., Perissocytheridea cribosa and Thalassocypria sp. Good indicators of alkaline waters are Paratanytarsus sp.1, Djalmabatista sp., Endotribelos sp., and Mastigodiaptomus nesus, whereas waters with $\mathrm{HCO}_{3}<275 \mathrm{mg} / \mathrm{L}$ were dominated by Stempellina sp., Tanytarsini J, K, Apedilum sp and Arctodiaptomus dorsalis.

Transfer functions that express quantitative relations between bioindicator species and environmental variables will ultimately be developed using results from this study. These transfer functions will be used to make quantitative paleoenvironmental inferences, by applying them to fossil assemblages in sediment cores retrieved from lakes in the region. Despite the utility of the collected data, additional sampling campaigns in aquatic ecosystems throughout Mexico, Guatemala, Belize and other parts of Central America are required. We also recommend return visits to previously studied ecosystems to capture seasonal variability. 


\section{ACKNOWLEDGMENTS}

We are grateful to the agencies and people who helped us with field and laboratory work, including the University of Belize, Forestry and Fisheries Departments (Belize), Universidad del Valle de Guatemala, CONAP, AMSCLAE, AMPI, FINABECE, Trifinio (Guatemala), SRE, CONAPESCA, ECOSUR-Chetumal (Mexico), Institut für Geosysteme und Bioindikation (Germany), TU-Braunschweig (Germany), Dietmar Keyser, Dustin Grzesik, Jason Curtis, David Klassen, José Harders, Carmen Herold, Bessie Oliva, Roberto Moreno, Eleonor de Tott, Margaret Dix, Margarita Palmieri, Alma Quilo, Gabriela Alfaro, Jacobo Blijdenstein, Melisa Orozco, Silja Ramirez, Wolfgang Riss, Evgenia Vinogradova, Luis Toruño, Mario Cruz, Rita Bugja, Luciana Mitsue, Susanne Krueger, Javier Pérez y Pérez, and Carolina Alvarado de Pérez. Special thanks to anonymous reviewers for detailed suggestions and comments. We are grateful for financial support provided by the Deutsche Forschungsgemeinschaft (DFG, grant Schw 671/3) and start-up money to A.S. provided by the TU Braunschweig.

\section{RESUMEN}

Los quironómidos, diatomeas y microcrustaceos que habitan ecosistemas acuáticos en el norte de los Neotrópicos son abundantes y diversos. Algunas especies son altamente sensibles a cambios en la composición química del agua y en el estado trófico. Este estudio se realizó como el primer paso para desarrollar funciones de transferencia para inferir condiciones ambientales en el norte de las tierras bajas de los Neotrópicos. Es por esto que las abundancias de especies bioindicadoras se relacionaron con múltiples variables ambientales con el fin de explotar al máximo su uso como indicadores ambientales y paleoambientales. Recolectamos y analizamos muestras de agua y de sedimento superficial de 63 cuerpos de agua, ubicados a lo largo de un gradiente trófico y de gradientes marcados de altitud ( $\sim 0-1560$ m.s.n.m.) y de precipitación $(\sim 400-3$ 200mm/año), desde el NO de la Península de Yucatán (México) hasta el sur de Guatemala. Relacionamos 14 variables limnológicas con las abundancias relativas de 282 especies de diatomeas, 66 morfoespecies de quironómidos, 51 especies de cladóceros, 29 especies de ostrácodos no-marinos y seis especies de agua dulce de cladóceros calanoides. La estadística multivariada indicó que el bicarbonato es el principal determinante de la distribución de quironómidos y copépodos. El estado trófico es el segundo factor más importante en determinar la distribución de quironómidos. La conductividad, que está relacionada con el gradiente de precipitación e influencia marina en la Península de Yucatán, es la principal variable en influir las comunidades de diatomeas, ostrácodos y cladóceros. Las diatomeas, quironómidos y cladóceros $(\mathrm{H}=2.4-2.6)$ presentaron diversidades más altas que los ostrácodos y copépodos $(\mathrm{H}=0.7-1.8)$. La riqueza de especies y la diversidad fueron más altas en las elevaciones bajas $(<450$ m.s.n.m) que en elevaciones altas en Guatemala. La distribución y diversidad de bioindicadores es afectada por múltiples factores incluyendo la altitud, precipitación, composición química del agua, estado trófico y el impacto humano.

Palabras clave: microcrustáceos, quironómidos, diatomeas, ecosistemas acuáticos, norte de los Neotrópicos, autecología, diversidad.

\section{REFERENCES}

Armitage, P.D., P.S. Cranston \& L.C.V. Pinder. 1995. The Chironomidae: biology and ecology of nonbiting midges. Chapman and Hall, London, England.

Battarbee, R.W., V.J. Jones, R.J. Flower, N.G. Cameron, H. Bennion, L. Cavalho \& S. Juggins. 2001. Diatoms, p. 155-202. In J.P. Smol, H.J.B. Birks \& W.M (eds.). Last. Tracking environmental change using lake sediments, vol 3. Kluwer Academic, Dordrecht, Netherlands.

Bennike, O. 1998. Fossil egg sacs of Diaptomus (Crustaceae: Copepoda) in Late Quaternary lake sediments. J. Paleolimnol. 19: 77-79.

Bowman, T.E. 1996. Freshwater calanoid copepods of the West Indies. Syllogeus 58: 237-246.

Brandorff, G.O. 2012. Distribution of some Calanoida (Crustacea, Copepoda) from the Yucatán Peninsula, Belize and Guatemala. Rev. Biol. Trop. 60: 187-202.

Brehm, V. 1939. La Fauna microscopica del Lago Petén, Guatemala. An. Esc. Nac. Cienc. Biol. 1: 173-203.

Brooks, S.J. \& H.J.B. Birks. 2001. Chironomid-inferred air temperatures from Lateglacial and Holocene sites in north-west Europe: progress and problems. Quat. Sci. Rev. 20: 1723-1741.

Casanova, S.M.C. \& R. Henry. 2004. Longitudial distribution of Copepoda populations in the transition zone of Paranapanema river and Jurumirim Reservoir (Sao Paulo, Brazil) and interchange with two later lakes. Braz. J. Biol. 64: 11-26.

Castellanos, E. \& M. Dix. 2009. Informe final, UVG, Levantamiento de la Línea Base del Lago de Atitlán Presentado al Ministerio de Ambiente y Recursos Naturales, Universidad del Valle de Guatemala, Guatemala. 
Cerny, M. \& P.D.N. Hebert. 1993. Genetic diversity and breeding system variation in Daphnia pulicaria from North American lakes. Heredity 71: 497-507.

Cohen, A. 2003. Paleolimnology. Oxford University, New York, USA.

De Eyto, E., K. Irvine \& G. Free. 2002. The use of Members of the Family Chydoridae (Anomopoda, Branchiopoda) as an Indicator of Lake Ecological Quality in Ireland. Biology and Environment. Proc. Roy. Ir. Acad. 102: 81-91.

De Oliveira Roque, F. \& S. Trivinho-Strixino. 2007. Chironomid species richness in low-order streams in the Brazilian Atlantic Forest: a first approximation through a Bayesian approach. J. N. Am. Benthol. Soc. 26: 221-231.

Dole-Olivier, M.J., D.M.P. Galassi, P. Marmonier \& M. Creuzé des Chatelliers. 2000. The biology and ecology of lotic microcrustaceans. Freshwater Biol. 44: 63-91.

Dudgeon, D., A.H. Arthington, M.O. Gessner, Z.I. Kawabata, D.J. Knowler, C. Lévêque, R.J. Naiman, A.H. Prieur-Richard, D. Soto \& M.L.J. Stiassny. 2006. Freshwater biodiversity: importance, threats, status and conservation challenges. Biol. Rev. 81: 163-182.

Eggermont, H., O. Heiri, J. Russell, M. Vuille M., L. Audenaert \& D. Verschuren. 2010. Paleotemperature reconstruction in tropical Africa using fossil Chironomidae (Insecta: Diptera). J. Paleolimnol. 43: 413-435.

Elías-Gutiérrez, M. 2006. Estudio comparativo del zooplancton en dos regiones de México. Informe final SNIB-CONABIO proyecto No. AS019. El Colegio de la Frontera Sur, Distrito Federal, México.

Elías-Gutiérrez, M., A.A. Kotov \& T. Garfias-Espejo. 2006. Cladocera (Crustacea: Ctenopoda, Anomopoda) from southern Mexico, Belize and Northern Guatemala. Zootaxa 119: 1-27.

Elías-Gutiérrez, M., E. Suárez-Morales, M.A. GutiérrezAguirre, M. Silva-Briano, J.G. Granados-Ramírez \& T. Garfias-Espejo. 2008. Cladocera y Copepoda de las aguas continentales de México. Universidad Nacional Autónoma de México, México.

Flössner, D. 2000. Die Haplopoda und Cladocera (ohne Bosminidae) Mitteleuropas. Backhuys, Leiden, Netherlands.

Fritz, S.C., S. Juggins, R.W. Battarbee \& D.R. Engstrom. 1991. Reconstruction of past changes in salinity and climate using a diatom-based transfer function. Nature 352: 706-708.

Furtos, N. 1933. The Ostracoda of Ohio. Ohio Biological Survey. The Ohio State University, Columbus, USA.

Furtos, N. 1936a. Fresh-water Ostracoda from Florida and North Carolina. Am. Mid. Nat. 17: 491-522.
Furtos, N. 1936b. On the Ostracoda from the cenotes of Yucatán and vicinity. The cenotes of Yucatan, a zoological and hydrographic survey. Carnegie Institution of Washington, Washington, USA.

Ghadouani, A., B.P. Alloul, Y. Zhang \& A.E.E. Prepas. 1998. Relationships between zooplankton community structure and phytoplankton in two lime treated eutrophic hardwater lakes. Freshwater Biol. 39: 775-790.

Gutiérrez-Aguirre, M.A. \& E. Suárez-Morales. 2000. New extension range of the diaptomid copepod Prionodiaptomus colombiensis Thiébaud, 1912 (Copepoda, Calanoida) with complementary description of this species. Zoosystema 22: 507-516.

Haberyan, K.A., S.P. Horn \& B.F. Cumming. 1997. Diatom assemblages from Costa Rican lakes: an initial ecological assessment. J. Paleolimnol. 17: 263-274.

Hausmann, S. \& F. Kienast. 2006. A diatom-inference model for nutrients screened to reduce the influence of background variables: Application to varved sediments of Greifensee and evaluation with measured data. Paleogeogr. Palaeoclimateol. Palaeoecol. 233: 96-112.

Hausmann, S. \& R. Pienitz. 2007. Seasonal climate inferences from high-resolution modern diatom data along a climate gradient: a case study. J. Paleolimnol. 38: 73-96.

Illyová, M. \& D. Némethová. 2005. Long-term changes in cladoceran assemblages in the Danube floodplain area (Slovak-Hungarian stretch). Limnologica-Ecol. Manag. Inland Waters 35: 274-282.

Keyser, D. 1976. Zur Kenntnis der brackigen mangrovebewachsenen Weichböden Südwest-Floridas unter besonderer Berücksitchtigung ihrer Ostracodenfauna. Ph.D. Thesis, Universität Hamburg, Hamburg, Germany.

Korovchinsky, N.M. 1992. Sididae and Holopediidae (Crustacea: Daphniiformes). SPB Academic, The Hague, Netherlands.

Kotov, A.A. \& P. Stifler. 2006. Cladocera family Ilyocryptidae (Branchiopoda: Cladocera: Anomopoda). Guide to the identification of the microinvertebrates of the Continental Waters of the World. Kenobi Productions, Ghent, Belgium and Backhuys, Leiden, Netherlands.

Krammer, K. \& H. Lange-Bertalot. 1986. Süsswasserflora von Mitteleuropa. Bd. 2/1. Bacillariophyceae: Naviculaceae. Gustav Fischer, Stuttgart, Germany.

Krammer, K. \& H. Lange-Bertalot. 1988. Süsswasserflora von Mitteleuropa. Bd. 2/2. Bacillariophyceae: Bacillariaceae, Epithmiaceae, Surirellaceae. Gustav Fischer, Stuttgart, Germany.

Krammer, K. \& H. Lange-Bertalot. 1991a. Süsswasserflora von Mitteleuropa. Bd. 2/3. Bacillariophyceae: 
Centrales, Fragilariaceae, Eunotiaceae. Gustav Fischer, Stuttgart, Germany.

Krammer, K. \& H. Lange-Bertalot. 1991b. Süsswasserflora von Mitteleuropa. Bd. 2/4. Bacillariophyceae: Achnanthaceae. Gustav Fischer, Stuttgart, Germany.

Krebs, C.J. 1989. Ecological Methodology. Harper and Row, New York, USA.

Lepš, J. \& P. Šmilauer. 2003. Multivariate analysis of ecological data using CANOCO. Cambridge University, Cambridge, England.

Lieder, U. 1996. Crustacea, Cladocera/Bosminidae. In J. Schwörbel, P. Zwick. Süßwasserfauna von Mitteleuropa 8/2-3. Gustav Fischer Verlag, Germany.

Lutz, W., L. Prieto \& W. Sanderson. 2000. Population, Development, and Environment on the Yucatán Peninsula: From Ancient Maya to 2030. International Institute for Applied Systems Analysis, Laxenburg, Austria.

Marrone, F., R. Barone \& L. Naselli-Flores. 2005. Cladocera (Branchiopoda: Anomopoda, Ctenopoda, and Onychopoda) from Sicilian Inland Waters: An Updated Inventory. Crustaceana 78: 1025-1039.

Massaferro, J., S. Ribeiro Guevara, A. Rizzo \& M. Arribére. 2004. Short-term environmental changes in Lake Morenito $\left(41^{\circ} \mathrm{S}, 71^{\circ} \mathrm{W}\right.$, Patagonia, Argentina) from analysis of sub-fossil chironomids. Aquat. Conserv. Mar. Freshwat. Ecosyst. 15: 23-30.

McKenzie, J.A. 1985. Carbon isotopes and productivity in the lacustrine and marine environment, p. 99-118. In W. Stumm. Chemical Processes in Lakes. John Wiley \& Sons, New York, USA.

Meisch, C. 2000. Freshwater Ostracoda of western and central Europe, In J. Schwörbel \& P. Zwick. Süßwasserfauna von Mitteleuropa. (8/1) Spektrum Akademischer GmbH, Germany.

Mischke, S., U. Herzschuh, G. Massmann \& C. Zhang. 2007. An ostracode conductivity transfer function for Tibetan lakes. J. Paleolimnol. 38: 509-524.

Moss, B., D. McKee, D. Atkinson, S.E. Collings, J.W. Eaton, A.B. Gill, I. Harvey, K. Hatton, T. Heyes \& D. Wilson. 2003. How important is climate? Effects of warming, nutrient addition and fish on phytoplankton in shallow lake microcosms. J. Appl. Ecol. 40: 782-792.

O'Sullivan, P.E. \& C.S. Reynolds. 2004. The lakes handbook. Limnology and limnetic ecology, vol 1. Blackwell, Cornwall, England.

Pérez, L., R. Bugja, J. Massaferro, P. Steeb, R. van Geldern, P. Frenzel, M. Brenner, B. Scharf \& A. Schwalb. 2010a. Post-Columbian environmental history of Lago Petén Itzá, Guatemala. Rev. Mex. Cienc. Geol. 27: 490-507.
Pérez, L., J. Lorenschat, M. Brenner, B. Scharf \& A. Schwalb. 2010b. Extant freshwater ostracodes (Crustacea: Ostracoda) from Lago Petén Itzá, Guatemala. Rev. Biol. Trop. 58: 871-895.

Pérez, L., J. Lorenschat, R. Bugja, M. Brenner, B. Scharf \& A. Schwalb. 2010c. Distribution, diversity and ecology of modern freshwater ostracodes (Crustacea), and hydrochemical characteristics of Lago Petén Itzá, Guatemala. J. Limnol. 69: 146-159.

Pérez, L., J. Lorenschat, R. Bugja, M. Brenner, P. Hoelzmann, G. Islebe, B. Scharf \& A. Schwalb. 2011a. Aquatic ecosystems of the Yucatán Peninsula and surrounding areas. Hydrobiologia 661: 407-433.

Pérez, L., P. Frenzel, M. Brenner, E. Escobar, P. Hoelzmann, B. Scharf \& A. Schwalb. 2011b. Late Quaternary (24-10 ka BP) environmental history of the Neotropical lowlands inferred from ostracodes in sediments of Lago Petén Itzá, Guatemala. J. Paleolimnol. 46: 59-74.

Perry, E.C., L. Marín, J. McClain \& G. Velázquez. 1995. Ring of cenotes (sinkholes), northwest Yucatan, Mexico: its hydrogeologic characteristics and possible association with the Chicxulub impact crater. Geology 23: 17-20.

Porinchu, D.F. \& G.M. MacDonald. 2003. The use and application of freshwater midges (Chironomidae: Insecta: Diptera) in geographical research. Prog. Phys. Geogr. 27: 378-422.

Rejmánková, E., J. Komárek, M. Dix, J. Komárková \& N. Girón. 2011. Cyanobacterial blooms in Lake Atitlan, Guatemala. Limnologica -Ecol. Manag. Inland Waters 41: 296-302.

Rivas Flores, A.W., R.E. Gómez Orellana \& A.J. Monterrosa Urías. 2010. Consideraciones generales para el estudio y monitoreo de diatomeas en los principales ríos de El Salvador. Formulación de una guía metodológica estandarizada para determinar la calidad ambiental de las aguas de los ríos de El Salvador, utilizando insectos acuáticos. Proyecto Universidad El Salvador (UES). Organización de los Estados Americanos (OEA), San Salvador, El Salvador.

Rosén, P., R. Hall, T. Korsman \& E. Renberg. 2000. Diatom transfer-functions for quantifying past air temperature, $\mathrm{pH}$ and total organic concentration from lakes in Northern Sweden. J. Paleolimnol. 24: 109-123.

Rosenmeier, M.F., M. Brenner, W.F. Kenney, T.J. Whitmore \& C.M. Taylor. 2004. Recent eutrophication in the southern basin of Lake Petén Itzá, Guatemala: human impact on a large tropical lake. Hydrobiologia 511:161-172.

Sala, O.E., F.S. III Chapin, J.J. Armesto, E. Berlow, J. Bloomfield, R. Dirzo, E. Huber-Sanwald, L.F. Huenneke, R.B. Jackson, A. Kinzig, R. Leemans, D.M. Lodge, H.A. Mooney, M. Oesterheld, N.L. Poff, M.T. Sykes, B.H. Walker, M. Walker \& D.H. Wall. 2000. 
Global biodiversity scenarios for the year 2100 . Science 287: 1770-1774.

Sánchez, M., J. Alcocer, E. Escobar \& A. Lugo. 2002. Phytoplankton of cenotes and anchialine caves along a distance gradient from the northeastern coast of Quintana Roo, Yucatan Peninsula. Hydrobiologia 467: 79-89.

Schmitter-Soto, J.J., F.A. Comín, E. Escobar-Briones, J. Herrera-Silveira, J. Alcocer, E. Suárez-Morales, M. Elías-Gutiérrez, V. Díaz-Arce, L.E. Marín \& B. Steinich. 2002. Hydrogeochemical and biological characteristics of cenotes in the Yucatan Peninsula (SE Mexico). Hydrobiologia 467: 215-228.

Schwalb, A. 2003. Lacustrine ostracodes as stable isotope recorders of late-glacial and Holocene environmental dynamics and climate. J. Paleolimnol. 29: 256-351.

Servicios Informativos y Publicitarios del Sureste (SIPSE). 2011. Se decretan tres áreas protegidas en Quintana Roo. SIPSE.COM, Campeche, Quintana Roo, Yucatán, México (Downloaded: November 10, 2011, http://www.sipse.com/noticias/96930--decretan-tresareas-protegidas-quintana-.html).

Sinev, A.Y. \& W. Hollwedel. 2002. Alona brandorffi sp. n. (Crustacea: Anomopoda: Chydoridae) a new species from Brazil, related to A. verrucosa Sars 1901. Hydrobiologia 472: 131-140.

Smirnov, N.N. 1992. Macrothricidae of the World. Guides to the Identification of the Macroinvertebrates of the Continental Waters of the World, vol. 1. SPB Acad. Publ., The Hague, Amsterdam, Netherlands.

Smirnov, N.N. 1996. Cladocera: the Chydorinae and Sayciinae (Chydoridae) of the World. Guides to the identification of the macroinvertebrates of the Continental Waters of the World, vol 11. SPB Acad. Publ., The Hague, Amsterdam, Netherlands.

Smith, A.J. 1993. Lacustrine ostracodes as hydrochemical indicators in lakes of the north-central United States. J. Paleolimnol. 8: 121-134.

Stich, H.B. \& G. Maier. 2007. Distribution and abundance of Daphnia pulicaria, a large Daphnia of the "pulex group", in Lake Constance (Lower Lake). Limnologica-Ecol. Manag. Inland Waters 37: 303-310.

Suárez-Morales, E. \& M. Elías-Gutiérrez. 2000. Two new Mastigodiaptomus (Copepoda, Diaptomidae) from southeastern Mexico, with a key for the identification of the known species of the genus. J. Nat. Hist. 34: 693-708.
Suárez-Morales, E. \& M. Elías-Gutiérrez. 2001. On the taxonomical status of Arctodiaptomus dampfi Brehm (Crustacea: Copepoda: Diaptomidae) with comments on A. dorsalis (Marsh). J. Limnol. 60: 11-18.

Suárez-Morales, E. 2003. Historical biogeography and distribution of the freshwater calanoid copepods (Crustacea: Copepoda) of the Yucatan Peninsula, Mexico. J. Biogeogr. 30: 1851-1859.

Suárez-Morales, E. \& J. Reid. 2003. An updated checklist of the continental copepod fauna of the Yucatan Peninsula, Mexico, with notes on its regional associations. Crustaceana 76: 977-991.

Swain, F.M. 1966. Bottom sediments of lake Nicaragua and Managua, western Nicaragua. J. Sediment. Petrol. 36: $522-540$.

Sylvestre, F. 2002. A high-resolution diatom reconstruction between 21,000 and $17,000{ }^{14} \mathrm{C}$ yr $\mathrm{BP}$ from the southern Bolivian Altiplano (18-23). J. Paleolimnol. 27: 45-57.

Ter Braak, C.J.F. \& P. Šmilauer. 2002. CANOCO 4.5. Microcomputer Power. Ithaca, New York, USA.

Vadadi-Fülöp, C. 2009. Zooplankton (Cladocera, Copepoda) dynamics in the River Danube upstream and downstream of Budapest, Hungary. Opusc. Zool. Budapest 40: 87-98.

Van Damme, K., A.Y. Sinev \& H.J. Dumont. 2011. Separation of Anthalona gen. n. from Alona Baird, 1843 (Branchiopoda: Cladocera: Anomopoda): morphology and evolution of scraping stenothermic alonines. Zootaxa 2875: 1-64.

Velez, M.I., J.H. Curtis, M. Brenner, J. Escobar, B.W. Leyden \& M. Popenoe de Hatch. 2011. Environmental and cultural changes in highland Guatemala inferred from Lake Amatitlán sediments. Geoarchaeol. 26: 346-364.

Vinogradova, E.M. \& H.W. Riss. 2007. Chironomids of the Yucatán Península. Chironomus 20: 32-35.

Walker, I.R., A.J. Levesque, L.C. Cwynar \& A.F. Lotter. 1997. An expanded surface-water palaeotemperature inference model for use with fossil midges from eastern Canada. J. Paleolimnol. 18: 165-178.

Walseng, B., D.O. Hessen, G. Halvorsen \& A.K. Schartau. 2006. Major contribution from littoral crustaceans to zooplankton species richness in lakes. Limnol. Oceanogr. 51: 2600-2606. 